\title{
The visual cortex as a crystal
}

\author{
Paul C. Bressloff ${ }^{\mathrm{a}, *}$, Jack D. Cowan ${ }^{\mathrm{b}}$ \\ a Department of Mathematics, University of Utah, Salt Lake City, UT 84112, USA \\ ${ }^{\mathrm{b}}$ Mathematics Department, University of Chicago, Chicago, IL 60637, USA
}

Received 15 May 2002; received in revised form 30 August 2002; accepted 4 September 2002

Communicated by J.P. Keener

\begin{abstract}
A theory of pattern formation in primary visual cortex (V1) is presented that takes into account its crystalline-like structure. The cortex is partitioned into fundamental domains or hypercolumns of a lattice describing the distribution of singularities or pinwheels in the orientation preference map. Each hypercolumn is modelled as a network of orientation and spatial frequency selective cells organised around a pair of pinwheels, which are associated with high and low spatial frequency domains, respectively. The network topology of the hypercolumn is taken to be a sphere with the pinwheels located at the poles of the sphere. Interactions between hypercolumns are mediated by anisotropic long-range lateral connections that link cells with similar feature preferences. Using weakly nonlinear analysis, we investigate the spontaneous formation of cortical activity patterns through the simultaneous breaking of an internal $\mathbf{O}(3)$ symmetry and a discrete lattice symmetry. The resulting patterns are characterised by states in which each hypercolumn exhibits a tuned response to both orientation and spatial frequency and the distribution of optimal responses across hypercolumns is doubly periodic or quasi-periodic with respect to the underlying lattice.
\end{abstract}

(C) 2002 Published by Elsevier Science B.V.

Keywords: Visual cortex; Horizontal connections; Hypercolumn; Spatial frequency; Pattern formation; Spherical symmetry; Planar lattice; Brillouin zone

\section{Introduction}

A major simplifying assumption in most large scale models of cortical tissue is that the interactions between cell populations are homogeneous and isotropic, that is, the pattern of connections is invariant under the Euclidean group $\mathbf{E}$ (2) of rotations, translations and reflections in the cortical plane (for a review see [16,22]). However, these assumptions are no longer valid when the detailed microstructure of cortex is taken into account. This is exemplified by the functional and anatomical organisation of primary visual cortex (V1) in cats and primates, which has a distinctly crystalline-like structure.

One of the basic functional properties of V1 is that cells signal topographically or retinotopically the location of a stimulus in the visual field. Superimposed upon this retinotopic map are a number of additional maps reflecting the fact that neurons respond preferentially to stimuli with particular features such as orientation, spatial frequency and left/right eye (ocular) dominance $[34,40,46]$. The maps of both ocular dominance and orientation preference

\footnotetext{
* Corresponding author.

E-mail address: bressloff@math.utah.edu (P.C. Bressloff).
} 


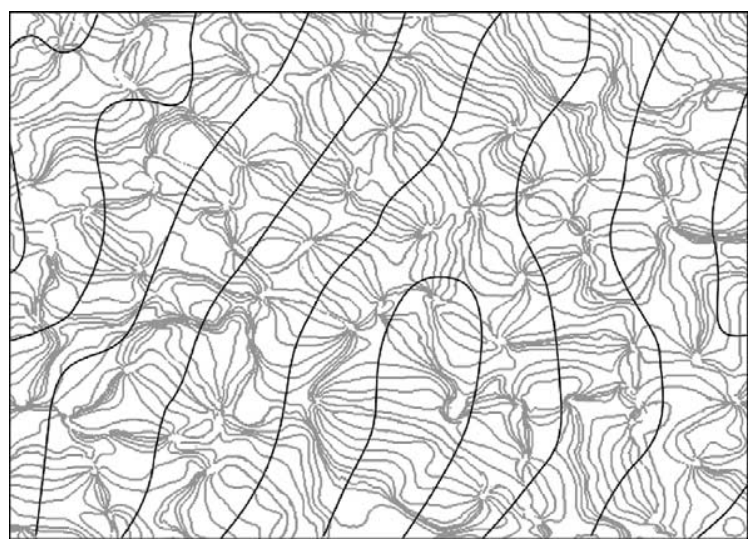

Fig. 1. Iso-orientation (light) and ocular dominance (dark) contours in a region of V1. Around each orientation pinwheel is a ring of orientation selective cells [13].

have been well characterised in cat and monkey, using a mixture of micro-electrode [27,28,30] and optical imaging techniques [5-7]. In particular, orientation preference appears to change continuously as a function of cortical location except at singularities or pinwheels where the scatter or rate of change of differing orientation preference labels is much higher (see Fig. 1), so that there is a weakening of orientation selectivity at the population level [39]. The distribution of spatial frequency preference across cortex is less clear. However, recent optical imaging data concerning spatial frequency maps in cat suggest that there is a correlation between the location of orientation preference pinwheels and regions selective for either low or high spatial frequencies [8,32]. Fig. 2 shows, for example, that some iso-orientation singularities are surrounded by regions of low spatial frequency preference located in a background of higher spatial frequency preferences.

Taken together these observations suggest that V1 has a crystalline-like structure in which various feature preference maps are organised around a lattice of orientation pinwheels. The existence of such a lattice naturally leads to a partitioning of cortex into fundamental domains or hypercolumns [29,34] that cover an approximate area of $1 \mathrm{~mm} \times 1 \mathrm{~mm}$ (in cats and primates) and include a full set or orientations and spatial frequencies per ocular dominance column. How does this crystalline-like structure of V1 manifest itself anatomically? Two cortical circuits

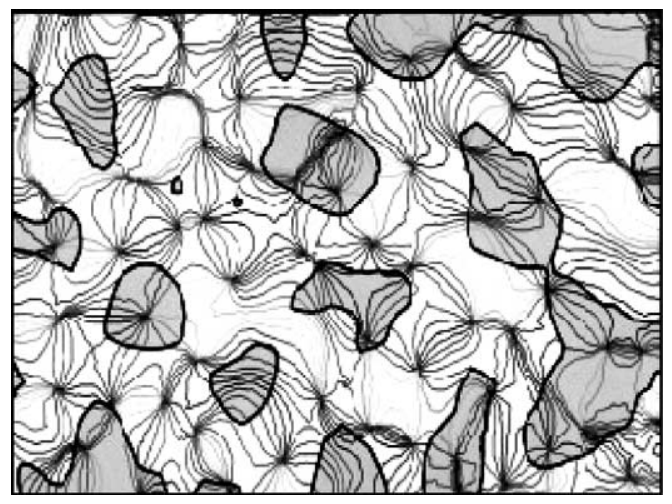

Fig. 2. Map of iso-orientation contours and low spatial frequency preferences in cat V1. Filled regions correspond to low spatial frequencies, unfilled to high. Redrawn from [8]. 
have been fairly well characterised. There is a local circuit operating at sub-hypercolumn dimensions consisting of a mixture of intra-cortical excitation and inhibition [20]. It has been suggested that such circuitry provides a substrate for the recurrent amplification and sharpening of the tuned response of cells to local visual stimuli. The best known example is the ring model of orientation preference and tuning [4,45], in which the local weights are assumed to vary as a function of the difference in orientation preference between the pre-synaptic and post-synaptic cells. One of the basic assumptions of the ring model (which has received recent experimental support [42]) is that the inhibitory connections are more broadly tuned with respect to orientation than the excitatory connections. The other circuit operates between hypercolumns, connecting cells with similar functional properties separated by several millimetres of cortical tissue. Optical imaging combined with labelling techniques has generated considerable information concerning the pattern of these long-range connections [5,6,10,36,49]. A particularly striking result concerns the intrinsic lateral connections in superficial layers of $\mathrm{V} 1$. The axons of these connections make terminal arbors only every $0.7 \mathrm{~mm}$ or so along their tracks [23,41] and they seem to connect mainly to cells with similar orientation preferences and ocularity [10,36,49]. In addition, there is a pronounced anisotropy in the pattern of such patchy connections - cells within differing hypercolumns tend to connect in directions parallel to their common preferred orientation $[10,36,49]$.

In this paper, we study pattern formation in a model of V1 that takes into account the crystalline structure described above. In particular, the cortex is partitioned into hypercolumns corresponding to the fundamental domains of a lattice of orientation pinwheels. Each hypercolumn is modelled as a network of interacting excitatory and inhibitory neural populations with orientation and spatial frequency preferences organised around a pair of pinwheels (see Section 2). Following our own recent work on orientation and spatial frequency tuning in a cortical hypercolumn $[14,15]$, the network topology of each hypercolumn is taken to be that of a sphere, with the poles of the sphere identified as low and high spatial frequency pinwheels, respectively. Assuming that the distribution of recurrent interactions within the network has internal $\mathbf{O}(3)$ symmetry (generalising the $\mathbf{O}(2)$ symmetry of the ring model [4]), we show how pattern formation on the sphere occurs via an $\mathbf{O}(3)$ symmetry breaking mechanism that excites the first few spherical harmonic components of the network activity profile and subsequently leads to the formation of a stable tuning surface whose solitary peak encodes the orientation and spatial frequency of the hypercolumn's response. Furthermore, the peak response can be locked to a weakly biased stimulus from the lateral geniculate nucleus (LGN).

We then introduce a model for the anisotropic lateral connections linking different hypercolumns on the lattice and highlight their symmetry properties (see Section 3). We show how such connections induce correlations between tuning surfaces of different hypercolumns through spontaneous breaking of both internal $\mathbf{O}(3)$ symmetry and the discrete lattice symmetry, leading to cortical patterns of activity that are doubly periodic (or quasi-periodic) with respect to the underlying lattice (Section 4). Our main result is to show that these activity patterns can select either an oriented pattern at intermediate spatial frequencies (which has two distinct forms), or else an unoriented pattern of alternating high and low spatial frequencies. This choice can be interpreted in terms of "two circuits" involving, respectively, the pinwheels and the linear zones of the orientation map. Moreover, changes in the spatial frequency dependence of the anisotropic lateral connections (perhaps mediated by neuromodulators) can switch between these different types of patterns. Interestingly, the basic patterns arising in the lattice model are consistent with those previously described in the continuous, uniform spatial frequency case [11]. However, there are two significant differences from our previous works. First, the inclusion of spatial frequency provides a more biologically plausible mechanism for selecting between oriented and non-oriented patterns. Second, Euclidean symmetry is explicitly broken by the existence of a physical lattice of orientation pinwheels. This means that doubly periodic patterns such as rolls, hexagons and squares arise naturally within our theory, rather than being imposed arbitrarily as a mathematical simplification. Differences between lattice and continuum models of V1 are discussed in Section 5. 


\section{A spherical model of a cortical hypercolumn}

In this section, we briefly review our spherical model of a cortical hypercolumn and show how orientation and spatial frequency tuning arise through spontaneous symmetry breaking of $\mathbf{O}(3)$ symmetry. Note that this model, which was first introduced in Ref. [14], actually has full $\mathbf{O}$ (3) symmetry and not just $\mathbf{S O}$ (3) symmetry as previously implied. Making such a distinction in this paper is important, since reflection symmetry will play a role in the coupled hypercolumn model (see Section 3). We have developed elsewhere a slightly different version of the spherical model, which involves linear-threshold rather than smooth sigmoid output functions [15]. The non-smooth version has the advantage that exact solutions for two-dimensional localised bumps (tuning surfaces) can be found using moment expansions along analogous lines to the ring model [4]. On the other hand, the weakly nonlinear analysis used to study the smooth version of the model is more easily extended to the coupled hypercolumn case (see Appendix A and B).

\subsection{Reduction of a hypercolumn to a sphere}

Motivated by the various optical imaging studies [5-8,31,32] cited in Section 1, we assume that the cortex can be partitioned into hypercolumns, each of which consists of orientation and spatial frequency selective neurons organised around a pair of pinwheels, as illustrated schematically in Fig. 3. Furthermore, we suppose that a local patch of (excitatory and inhibitory) cells within a given hypercolumn can be labelled by the pair of cortical labels $\{p, \phi\}$, where $p \in\left[p_{\mathrm{L}}, p_{\mathrm{H}}\right]$ denotes the spatial frequency preference and $\phi \in[0, \pi)$ the orientation preference of a cell. (For simplicity, we neglect ocular dominance here: this could be incorporated into the model by introducing an additional discrete label for left/right eye dominance.) Typically, the bandwidth of a hypercolumn is between three and four octaves, that is, $p_{\mathrm{H}} \approx 2^{n} p_{\mathrm{L}}$ with $n=3.5$. This is consistent with the observations of Hubel and Wiesel [29], who found a two octave scatter of receptive field sizes at each cortical location.

Rather than attempting to accurately model the distribution of orientation and spatial frequency preferences within a hypercolumn, we consider a reduced model in which only a subset of neurons are explicitly represented, with the remaining neurons acting as an effective background cell medium. That is, we consider the union $D_{\mathrm{L}} \cup D_{\mathrm{H}}$ of two disc regions enclosing the low $(\mathrm{L})$ and high $(\mathrm{H})$ spatial frequency pinwheels, respectively. (Note that the ring model $[4,45]$ effectively reduces a hypercolumn to an annular region of orientation selective cells surrounding a single pinwheel and having a fixed spatial frequency.) As a further simplification, suppose that interactions with the background cortical tissue leads to an effective identification or sewing together of the two disc boundaries $\partial D_{\mathrm{H}}$ and $\partial D_{\mathrm{L}}$. The resulting topological space is then a sphere. Identifying the north and south poles of the sphere with

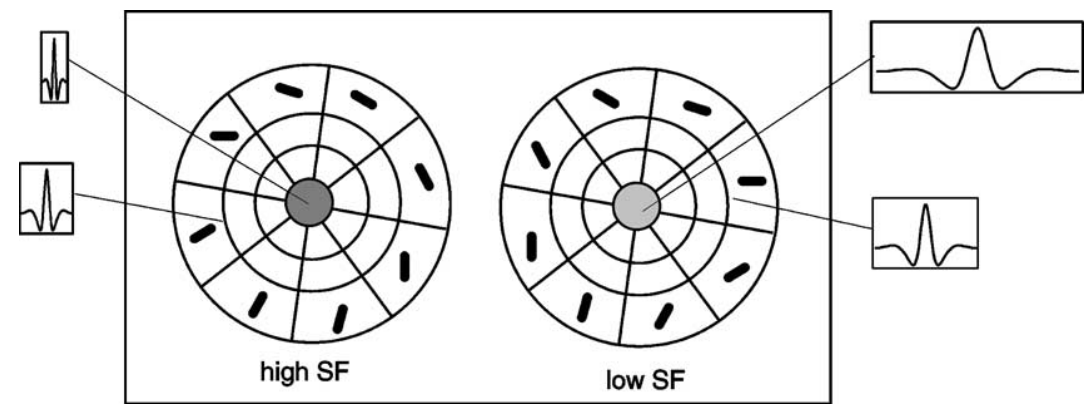

Fig. 3. Reduced model of a hypercolumn consisting of orientation and spatial frequency selective cells organised around a pair of pinwheels. One pinwheel is a site of high $(\mathrm{H})$ spatial frequencies and the other low $(\mathrm{L})$ spatial frequencies. The hypercolumn is represented as a pair of disc regions $D_{\mathrm{H}}, D_{\mathrm{L}}$ enclosing the two pinwheels, surrounded by a background of intervening neurons. 


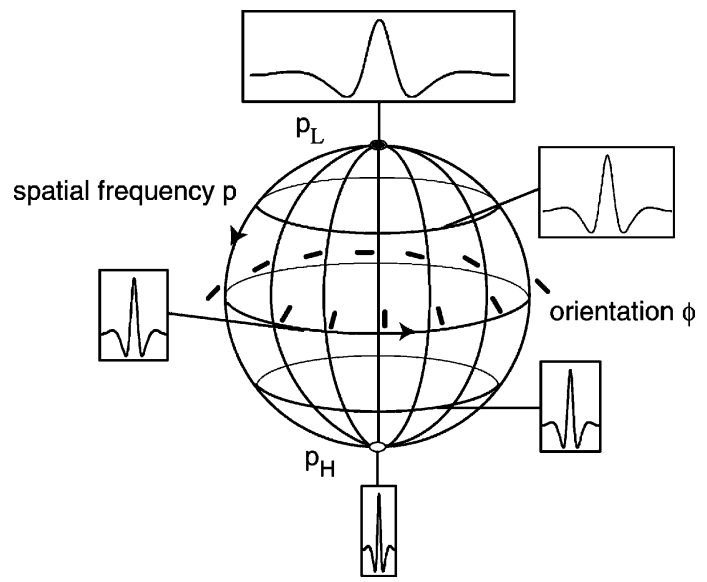

Fig. 4. Spherical network topology. Orientation and spatial frequency labels are denoted by $(\phi, p)$ with $0 \leq \phi<\pi$ and $p_{\mathrm{L}} \leq p \leq p_{\mathrm{H}}$.

the low and high spatial frequency pinwheels leads to a spherical model of a cortical hypercolumn [14,15], see Fig. 4. It is important to emphasise that the spherical topology is a mathematical idealisation of the effective weight pattern of local interactions within a hypercolumn and does not correspond to the actual distribution of cells in the cortical plane. It turns out that representing the hypercolumn as a sphere has a number of desirable consequences [15]. In particular, the associated dynamical model reproduces a number of experimental observations regarding correlations between spatial frequency and orientation tuning curves [32,37,38]. One particular example is the reduction in orientation selectivity at high and low spatial frequency pinwheels (see Section 2.3).

Taking $(\theta, \phi)$ to be the angular coordinates on the sphere with $\theta \in[0, \pi), \phi \in[0, \pi)$, we set

$$
\theta \equiv \mathcal{Q}(p)=\pi \frac{\log \left(p / p_{\mathrm{L}}\right)}{\log \left(p_{\mathrm{H}} / p_{\mathrm{L}}\right)} .
$$

That is, $\theta$ varies linearly with $\log p$. This is consistent with experimental data that suggests a linear variation of $\log p$ with cortical separation [32]. Let $a(\theta, \phi, t)$ denote the activity of a population of cells on the sphere with spatial frequency label given by Eq. (2.1) and suppose that $a$ evolves according to the equation [14]

$$
\frac{\partial a(\theta, \phi, t)}{\partial t}=-a(\theta, \phi, t)+\int_{0}^{\pi} \int_{0}^{\pi} W\left(\theta, \phi \mid \theta^{\prime}, \phi^{\prime}\right) \sigma\left[a\left(\theta^{\prime}, \phi^{\prime}, t\right)\right] \mathcal{D}\left(\theta^{\prime}, \phi^{\prime}\right)+h(\theta, \phi),
$$

where $\mathcal{D}(\theta, \phi)=\sin \theta \mathrm{d} \theta \mathrm{d} \phi / 2 \pi$ is the integration measure on the sphere. Here $W$ represents the distribution of recurrent interactions within the hypercolumn, $h(\theta, \phi)$ is a weakly biased stimulus from the LGN and $\sigma[a]$ is the smooth nonlinear sigmoid function

$$
\sigma[a]=\frac{\sigma_{\max }}{1+\mathrm{e}^{-g\left(a-a_{\mathrm{th}}\right)}}
$$

for constant gain $g$ and threshold $a_{\text {th }}$. We have also fixed the unit of time to be of the order $10 \mathrm{~ms}$ by setting the membrane time constant to unity.

\section{2. $\mathbf{O}(3)$-invariant weight distribution and spherical harmonics}

Given a spherical topology, it is natural to construct a local weight distribution that is invariant with respect to coordinate rotations and reflections of the sphere, that is, the symmetry group $\mathbf{O}(3)$. This spherical symmetry, 

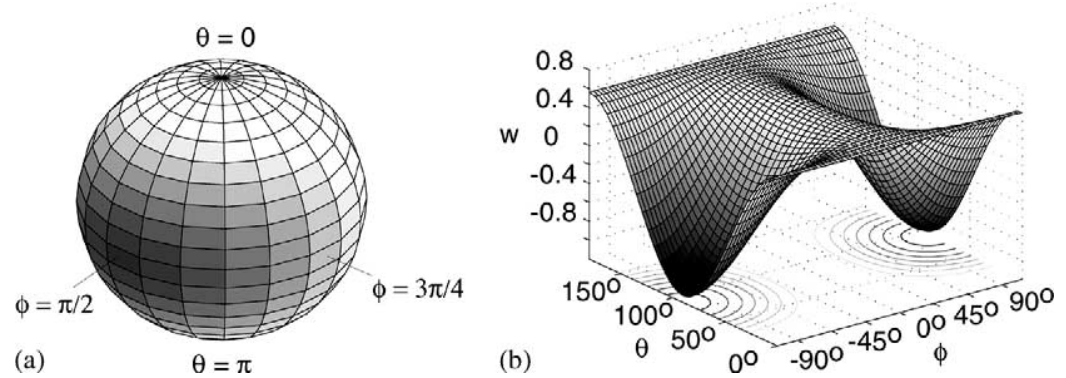

Fig. 5. Two-dimensional plot of $W\left(\theta, \phi \mid \theta^{\prime}, \phi^{\prime}\right)$ given by the $\mathbf{O}$ (3)-invariant weight distribution (2.5) with $\tilde{W}_{0}=-1$ and $\tilde{W}_{1}=1$. We set $\phi^{\prime}=0$, $\theta^{\prime}=\theta$ and plot $w$ as a function of $\theta$ and $\phi$. (a) Contour plot of $w$ on the sphere with light and dark regions correspond to excitation and inhibition, respectively. (b) Surface plot of $w$ in the $(\theta, \phi)$-plane.

which generalises the $\mathbf{O}(2)$ circular symmetry of the ring model, implies that the pattern of connections within the hypercolumn depends only on the relative distance of cells on the sphere as determined by their angular separation along geodesics or great circles. That is, given two points on the sphere $(\theta, \phi)$ and $\left(\theta^{\prime}, \phi^{\prime}\right)$ their angular separation $\alpha$ is determined from the equation

$$
\cos \alpha=\cos \theta \cos \theta^{\prime}+\sin \theta \sin \theta^{\prime} \cos \left(2\left[\phi-\phi^{\prime}\right]\right) .
$$

This suggests that the simplest non-trivial form for the local weight distribution is

$$
W\left(\theta, \phi \mid \theta^{\prime}, \phi^{\prime}\right)=\tilde{W}_{0}+\tilde{W}_{1}\left(\cos \theta \cos \theta^{\prime}+\sin \theta \sin \theta^{\prime} \cos \left(2\left[\phi-\phi^{\prime}\right]\right)\right) .
$$

In Fig. 5, we plot $W\left(\theta, \phi \mid \theta^{\prime}, \phi^{\prime}\right)$ as a function of $(\theta, \phi)$ for $\theta^{\prime}=\theta, \phi^{\prime}=0$ and $\tilde{W}_{1}>\tilde{W}_{0}$. It can be seen that away from the pinwheels (poles of the sphere at $\theta=0, \pi$ ), cells with similar orientation excite each other, whereas those with differing orientation inhibit each other. This is the standard interaction assumption of the ring model $[4,42,45]$. On the other hand, around the pinwheels, all orientations uniformly excite, which is consistent with the fact that although the cells around a pinwheel can differ greatly in their orientation preference, they are physically close together within the hypercolumn.

It is possible to construct a more general $\mathbf{O}(3)$-invariant weight distribution using spherical harmonics. Any sufficiently smooth function $f(\theta, \phi)$ on the sphere can be expanded in a uniformly convergent double series of spherical harmonics

$$
f(\theta, \phi)=\sum_{n=0}^{\infty} \sum_{m=-n}^{n} a_{n m} Y_{n}^{m}(\theta, \phi) .
$$

The functions $Y_{n}^{m}(\theta, \phi)$ constitute the angular part of the solutions of Laplace's equation in three dimensions and thus form a complete orthonormal set. The orthogonality relation is

$$
\int_{0}^{\pi} \int_{0}^{\pi} Y_{n_{1}}^{m_{1} *}(\theta, \phi) Y_{n_{2}}^{m_{2}}(\theta, \phi) \mathcal{D}(\theta, \phi)=\frac{1}{4 \pi} \delta_{n_{1}, n_{2}} \delta_{m_{1}, m_{2}} .
$$

The spherical harmonics are given explicitly by

$$
Y_{n}^{m}(\theta, \phi)=\sqrt{\frac{2 n+1}{4 \pi} \frac{(n-m) !}{(n+m) !}} P_{n}^{m}(\cos \theta) \mathrm{e}^{2 \mathrm{i} m \phi}
$$

for $n \geq 0$ and $-n \leq m \leq n$, where $P_{n}^{m}(\cos \theta)$ is an associated Legendre function. The lowest order spherical harmonics (which will play an important role later) are shown in Table 1. (Note that we have adjusted the definition 
Table 1

Spherical harmonics up to second order

\begin{tabular}{lll}
\hline & $m=0$ & $m= \pm 1$ \\
\hline$l=0$ & $Y_{0}^{0}(\theta, \phi)=\frac{1}{\sqrt{4 \pi}}$ & $Y_{1}^{ \pm 1}(\theta, \phi)=\sqrt{\frac{3}{8 \pi} \sin \theta \mathrm{e}^{ \pm 2 \mathrm{i} \phi}}$ \\
$l=1$ & $Y_{1}^{0}(\theta, \phi)=\sqrt{\frac{3}{4 \pi}} \cos \theta$ & $Y_{2}^{ \pm 1}(\theta, \phi)=\sqrt{\frac{45}{96 \pi} \sin 2 \theta \mathrm{e}^{ \pm 2 \mathrm{i} \phi}}$ \\
$l=2$ & $Y_{2}^{0}(\theta, \phi)=\sqrt{\frac{45}{16 \pi}}\left(\cos ^{2} \theta-\frac{1}{3}\right)$ & $Y_{2}^{ \pm 2}(\theta, \phi)=\sqrt{\frac{45}{96 \pi}} \sin ^{2} \theta \mathrm{e}^{ \pm 4 \mathrm{i} \phi}$
\end{tabular}

of the spherical harmonics to take into account the fact that $\phi$ takes values between 0 and $\pi$.) The action of $\mathbf{S O}(3)$ on $Y_{n}^{m}(\theta, \phi)$ involves $(2 n+1) \times(2 n+1)$ unitary matrices associated with irreducible representations of $S U(2)$ [2]. From the unitarity of these representations, one can construct an SO(3)-invariant weight distribution of the general form

$$
W\left(\theta, \phi \mid \theta^{\prime}, \phi^{\prime}\right)=4 \pi \sum_{n=0}^{\infty} \tilde{W}_{n} \sum_{m=-n}^{n} Y_{n}^{m^{*}}\left(\theta^{\prime}, \phi^{\prime}\right) Y_{n}^{m}(\theta, \phi)
$$

with $\tilde{W}_{n}$ real. It is simple to check that this weight distribution is also invariant under $\left(\phi, \phi^{\prime}\right) \rightarrow\left(-\phi,-\phi^{\prime}\right)$ so that it is actually $\mathbf{O}(3)$-invariant. For simplicity, we neglect higher harmonic contributions to $W\left(P \mid P^{\prime}\right)$ by setting $\tilde{W}_{n}=0$ for $n \geq 2$ so that Eq. (2.9) reduces to Eq. (2.5) on rescaling $\tilde{W}_{1}$.

\subsection{Amplitude equation for orientation and spatial frequency tuning}

We now show how sharpened orientation and spatial frequency tuning can occur through spontaneous symmetry breaking of $\mathbf{O}(3)$. Assume, for the moment, that the stimulus from the LGN is uniform (with $h(\theta, \phi)=\bar{h}$ ) such that Eq. (2.2) has $a(\theta, \phi)=\bar{a}$ as a fixed point solution. Linearising about the fixed point and setting $a(\theta, \phi, t)=$ $\bar{a}+\mathrm{e}^{\lambda t} u(\theta, \phi)$ leads to an eigenvalue equation for the linear eigenmodes $u(\theta, \phi)$ :

$$
\lambda u(\theta, \phi)=-u(\theta, \phi)+\mu \int_{0}^{\pi} \int_{0}^{\pi} W\left(\theta, \phi \mid \theta^{\prime}, \phi^{\prime}\right) u\left(\theta^{\prime}, \phi^{\prime}\right) \mathcal{D}\left(\theta^{\prime}, \phi^{\prime}\right)
$$

(with a factor $\sigma^{\prime}(\bar{a})$ absorbed into $\mu$ ). Substituting the distribution (2.9) for $W$ and using the orthogonality relation (2.7) shows that the linear eigenmodes are spherical harmonics with $\lambda=\lambda_{n} \equiv-1+\mu \tilde{W}_{n}$ for $u(\theta, \phi)=Y_{n}^{m}(\theta, \phi)$, $-n \leq m \leq n$. Thus the $n$th eigenvalue is $(2 n+1)$-fold degenerate and the associated (real) eigenmodes can be written in the general form $u_{0}(\theta, \phi)=z_{0} Y_{0}^{0}(\theta, \phi)$ and

$$
u_{n}(\theta, \phi)=z_{0} Y_{n}^{0}(\theta, \phi)+\sum_{m=1}^{n}\left[z_{m} Y_{n}^{m}(\theta, \phi)+z_{m^{*}} Y_{n}^{m^{*}}(\theta, \phi)\right]
$$

for $n \geq 1$ and $z_{0}$ real. Suppose that $\tilde{W}_{1}>\tilde{W}_{n}$ for all $n \neq 1$. The fixed point $a=\bar{a}$ then destabilises at a critical value of the coupling $\mu_{\mathrm{c}}=1 / \tilde{W}_{1}$ due to excitation of the first order spherical harmonics. Sufficiently close to the bifurcation point, the resulting activity profile $u=a-\bar{a}$ can be written in the form (see Table 1)

$$
u(\theta, \phi, t)=\sum_{m=0, \pm 1} c_{m}(t) f_{m}(\theta, \phi)
$$

for real coefficients $c_{0}, c_{ \pm}$and

$$
f_{0}(\theta, \phi)=\cos \theta, \quad f_{1}(\theta, \phi)=\sin \theta \cos 2 \phi, \quad f_{-1}(\theta, \phi)=\sin \theta \sin 2 \phi .
$$


Amplitude equations for these coefficients can be obtained by carrying out a perturbation expansion of Eq. (2.2) with respect to the small parameter $\varepsilon=\mu-\mu_{\mathrm{c}}$. As shown in the appendix, this leads to the Stuart-Landau equations

$$
\frac{\mathrm{d} c_{m}}{\mathrm{~d} t}=c_{m}\left(\frac{\mu-\mu_{\mathrm{c}}}{\mu_{\mathrm{c}}}-\mathcal{W} \sum_{p=0, \pm 1} c_{p}^{2}\right)
$$

where

$$
\mathcal{W}=-\left[\frac{3 \sigma_{3}}{5}+\frac{2 \sigma_{2}^{2}}{3} \frac{\mu_{\mathrm{c}} \tilde{W}_{0}}{1-\mu_{\mathrm{c}} \tilde{W}_{0}}+\frac{8 \sigma_{2}^{2}}{15} \frac{\mu_{\mathrm{c}} \tilde{W}_{2}}{1-\mu_{\mathrm{c}} \tilde{W}_{2}}\right] .
$$

Here $\sigma_{2}, \sigma_{3}$ are coefficients in the Taylor expansion of the firing rate function $\sigma$,

$$
\sigma(a)=\sigma(\bar{a})+\sigma^{\prime}(\bar{a})\left[(a-\bar{a})+\sigma_{2}(a-\bar{a})^{2}+\sigma_{3}(a-\bar{a})^{3}+\cdots\right]
$$

and $\tilde{W}_{0}, \tilde{W}_{2}$ are the zeroth and second order coefficients in the spherical harmonic expansion of the $\mathbf{O}(3)$-invariant weight distribution (2.9).

It is clear that the amplitude equations are equivariant with respect to the action of the orthogonal group $\mathbf{O}(3)$ on $\left(c_{0}, c_{+}, c_{-}\right)$, which reflects the underlying spherical symmetry. Moreover, defining

$$
R=\sum_{m=0, \pm 1} c_{m}^{2}
$$

we see that

$$
\frac{\mathrm{d} R}{\mathrm{~d} t}=2 R\left(\frac{\mu-\mu_{\mathrm{c}}}{\mu_{\mathrm{c}}}-\mathcal{W} R\right)
$$

which has a stable fixed point at $R_{0}=\left(\mu-\mu_{\mathrm{c}}\right) /\left[\mu_{\mathrm{c}} \mathcal{W}\right]$, provided that $\mathcal{W}>0$. This corresponds to an $\mathbf{O}(3)$-invariant submanifold of marginally stable states

$$
u(\theta, \phi)=\sum_{m=0, \pm 1} c_{m} f_{m}(\theta, \phi), \quad \sum_{m=0, \pm 1} c_{m}^{2}=R_{0}
$$

Eq. (2.19) represents a tuning surface for orientation and spatial frequency with a solitary peak whose location is determined by the values of the coefficients $\left(c_{0}, c_{1}, c_{-1}\right)$. Such a solution spontaneously breaks the underlying $\mathbf{O}$ (3) symmetry. However, full spherical symmetry is recovered by noting that rotation or reflection of the solution corresponds to an orthogonal transformation of the coefficients $c_{0}, c_{ \pm 1}$. Thus the action of $\mathbf{O}(3)$ is to shift the location of the peak of the activity profile on the sphere, that is, to change the particular orientation and spatial frequency selected by the tuning surface.

Now suppose that there exists a weakly biased, time-independent stimulus from the LGN of the form

$$
h(\theta, \phi)=\bar{h}+\delta h[\cos \theta \cos \Theta+\sin \theta \sin \Theta \cos (2[\phi-\Phi])] .
$$

Eq. (2.20) represents a unimodal function on the sphere with a single peak at $\{\Theta, \Phi\}$. Here we ignore higher order spherical harmonic contributions to the LGN stimulus, since the pattern forming instability amplifies only the first order harmonic components-it is these components that couple to the cubic amplitude equation. The effects of a weakly biased LGN stimulus can be studied within the framework of the amplitude equation approach by taking $\delta h=\mathcal{O}\left(\varepsilon^{3 / 2}\right)$. The stimulus (2.20) then generates an additional contribution to the cubic amplitude equation that explicitly breaks the hidden $\mathbf{O}(3)$ symmetry:

$$
\frac{\mathrm{d} c_{m}}{\mathrm{~d} t}=c_{m}\left(\frac{\mu-\mu_{\mathrm{c}}}{\mu_{\mathrm{c}}}-\mathcal{W} \sum_{p=0, \pm 1} c_{p}^{2}\right)+\delta h f_{m}(\Theta, \Phi), \quad m=0, \pm 1 .
$$


Eq. (2.21) has fixed point solutions of the form $c_{m}=C f_{m}$ with $C$ a root of the cubic equation

$$
C\left(\frac{\mu-\mu_{\mathrm{c}}}{\mu_{\mathrm{c}}}-\mathcal{W} C^{2}\right)+\delta h=0 .
$$

If $\mathcal{W}>0$, then there exists a single stable branch along which the peak of the tuning surface becomes locked to the stimulus. To a first order approximation, the resulting amplitude is of the form

$$
u(\theta, \phi)=C[\cos \theta \cos \Theta+\sin \theta \sin \Theta \cos (2[\phi-\Phi])] .
$$

Hence, the peak of the tuning surface is located at $\{\Theta, \Phi\}$. Since $h_{m}=\mathcal{O}\left(\varepsilon^{3 / 2}\right)$ whereas $c_{m}=\mathcal{O}\left(\varepsilon^{1 / 2}\right)$, we see that the cortical model acts as an amplifier for the first order spherical harmonic components of the weakly biased stimulus from the LGN. As the amplitude of the solution increases away from the bifurcation point, higher order harmonics will also be excited. Nevertheless, the first order harmonics will still be dominant so that Eq. (2.23) remains a reasonable first approximation of the tuning surface.

\subsection{Numerical calculations}

Eq. (2.2) can be solved numerically using the discretisation scheme introduced by [48] in their study of pattern formation for a reaction-diffusion system on a sphere. The sphere is discretised with a grid of $M \times N$ sites so that $\Delta \theta=\pi / M$ and $\Delta \phi=\pi / N$. In order to avoid the singularities at $\theta=0, \pi$, we take discretised coordinates

$$
\theta_{m}=(m-1 / 2) \Delta \theta, \quad m=1, \ldots, M, \quad \phi_{n}=n \Delta \phi, \quad n=1, \ldots, N .
$$

The weight distribution (2.5) naturally incorporates the topology of the sphere in which (i) the nearest neighbour of the point $(m, N)$ is $(m, 0)$ for $m=1, \ldots, M$, (ii) the nearest neighbour of the point $(1, n)$ is $(1, n+N / 2)$ for $n=1, \ldots, N / 2$ (that is, close to the $\theta=0$ singularity) and (iii) the nearest neighbour of the point $(M, n)$ is $(M, n+N / 2)$ for $n=1, \ldots, N / 2$ (that is, close to the $\theta=\pi$ singularity). In our numerical calculations, we use a grid of size $M=16$ and $N=32$ and use a low order Runge-Kutta method to solve the resulting system of coupled ODEs. Results are shown in Figs. 6 and 7.

A useful representation of the network response is to project the tuning surface on to the $(p, \phi)$-plane. This is illustrated in Fig. 6(a) for $\Theta=\pi / 2$ (corresponding to an intermediate stimulus frequency $p=2 c /^{\circ}$ ) and $\Phi=90^{\circ}$. In Fig. 6(b), we plot the response as a function of spatial frequency at the optimal orientation for various stimulus amplitudes $\delta h$. The height of the spatial frequency tuning curves increases with the stimulus amplitude $\delta h$, but the width at half height is approximately the same (as can be checked by rescaling the tuning curves to the same height). Since $\delta h$ increases with the contrast of a stimulus, this shows that the network naturally exhibits contrast invariance. Corresponding orientation tuning curves are shown in Fig. 6(c) and are also found to exhibit contrast invariance.

Note that projecting the spherical tuning surface on to the $\{p, \phi\}$-plane breaks the underlying $\mathbf{O}(3)$ symmetry of the sphere. Consequently, the shape of the planar tuning surface is not invariant with respect to shifts in the location of the peak of the tuning surface, see Fig. 7(a). The non-invariance of the planar tuning surface is related to the fact that solutions of the spherical model are not separable with respect to orientation and spatial frequency-the activity profile cannot be written in the form $u(\theta, \phi)=U(\theta) V(\phi)$. The resulting distortions generate behaviour that is consistent with some recent experimental observations. (a) At low and high spatial frequencies (that is, towards the pinwheels), there is a broadening of the tuned response to orientation, see Fig. 7(b). In our model, the reduction of orientation selectivity around the pinwheels is an aggregate property of the local population of cells. It has been found experimentally that individual neurons close to pinwheels are actually orientation selective [39], but there is a broad distribution of orientation preferences within the pinwheel region so that the average response of the population is only weakly orientation selective. (b) There is a systematic shift in the peak of spatial 

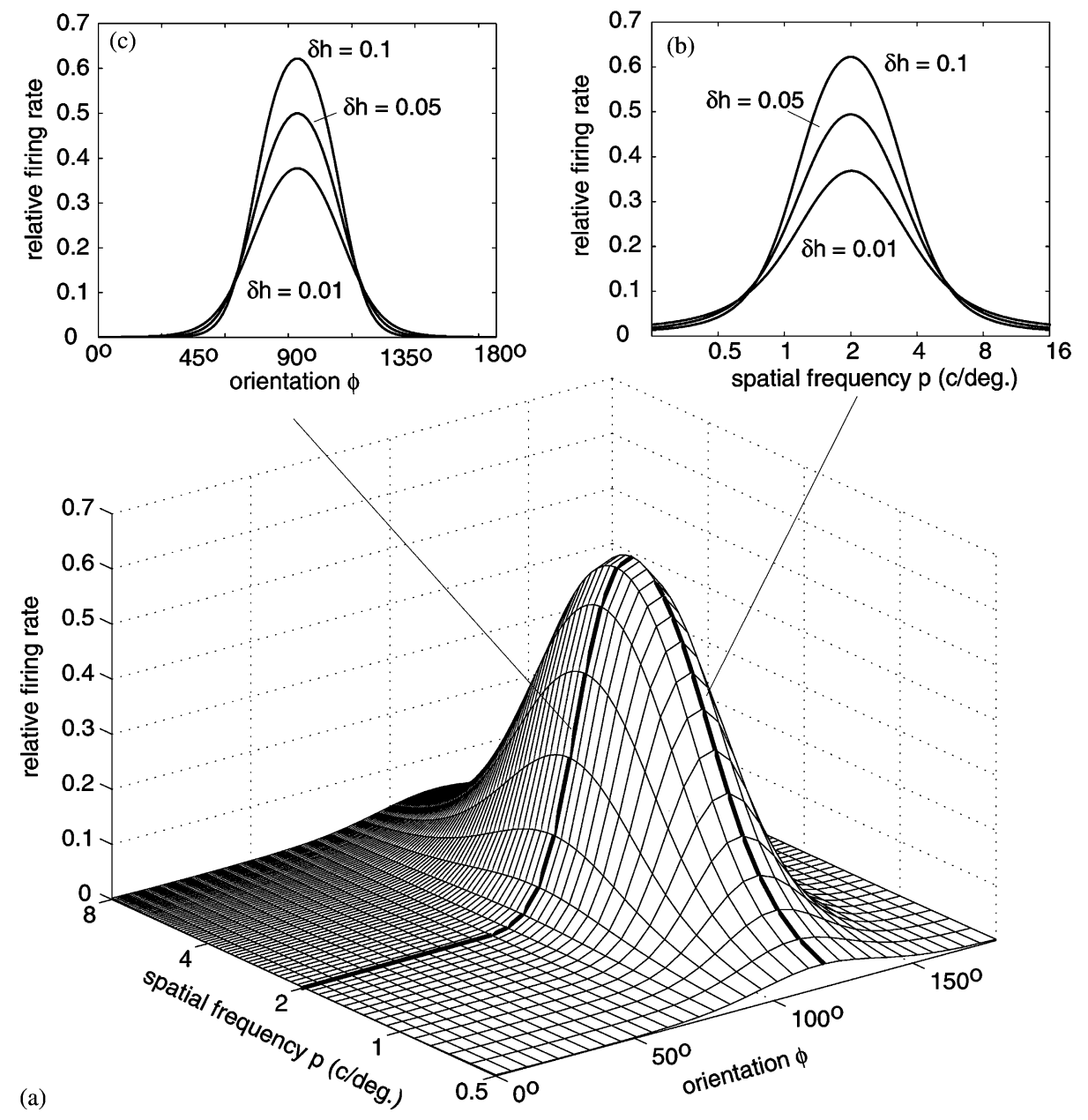

Fig. 6. Plot of normalised firing rate $\sigma(a) / \sigma_{\max }$ in response to a weakly biased stimulus from the LGN with $\Theta=\pi / 2$ and $\Phi=90^{\circ}$. The firing rate function (2.3) has a gain $g=5$, a threshold $a_{\text {th }}=0.6$ and $\bar{a}=0$. The weight coefficients are $\tilde{W}_{0}=-2, \tilde{W}_{1}=1, \tilde{W}_{n}=0, n>1$. The range of spatial frequencies is $p_{\mathrm{L}} \leq p \leq p_{\mathrm{H}}$ with $p_{\mathrm{L}}=0.5 c / \mathrm{deg}$ and $p_{\mathrm{H}}=8 c / \mathrm{deg}$. (a) Tuning surface in the $\{p, \phi\}$-plane. (b) Response as a function of spatial frequency $p$ at the optimal orientation $\phi=\Phi$. (c) Response as a function of orientation $\phi$ at the optimal spatial frequency $p=\mathcal{Q}^{-1}(\theta)$.

frequency tuning curves at non-optimal orientations that is towards the spatial frequency of the closest pinwheel, see Fig. 7(c). There is some suggestion of spatial frequency shifts in recent optical imaging data, supporting the notion of non-separability [32].

It turns out that there is a non-trivial relationship between the optimal spatial frequency of the cortical response as determined by $\Theta$ and the actual spatial frequency $p_{\mathrm{s}}$ of an external visual stimulus [15]. The transformation from visual to cortical stimulus can be described in terms of a convolution with respect to some feedforward receptive field [25]. If the low order spherical harmonic components of the resulting feedforward stimulus (see Eq. (2.20)) are then amplified by recurrent interactions, the optimal spatial frequency is shifted relative to the stimulus frequency, that is, $\Theta \neq \mathcal{Q}\left(p_{\mathrm{s}}\right)$; such a mismatch can be eliminated by feedback from cortex to LGN [15]. This issue is not so relevant here, however, since we are concerned with the case of spontaneous pattern formation in the absence of LGN modulation. The location $(\Theta, \Phi)$ of the peak response is then arbitrary for an isolated hypercolumn. 

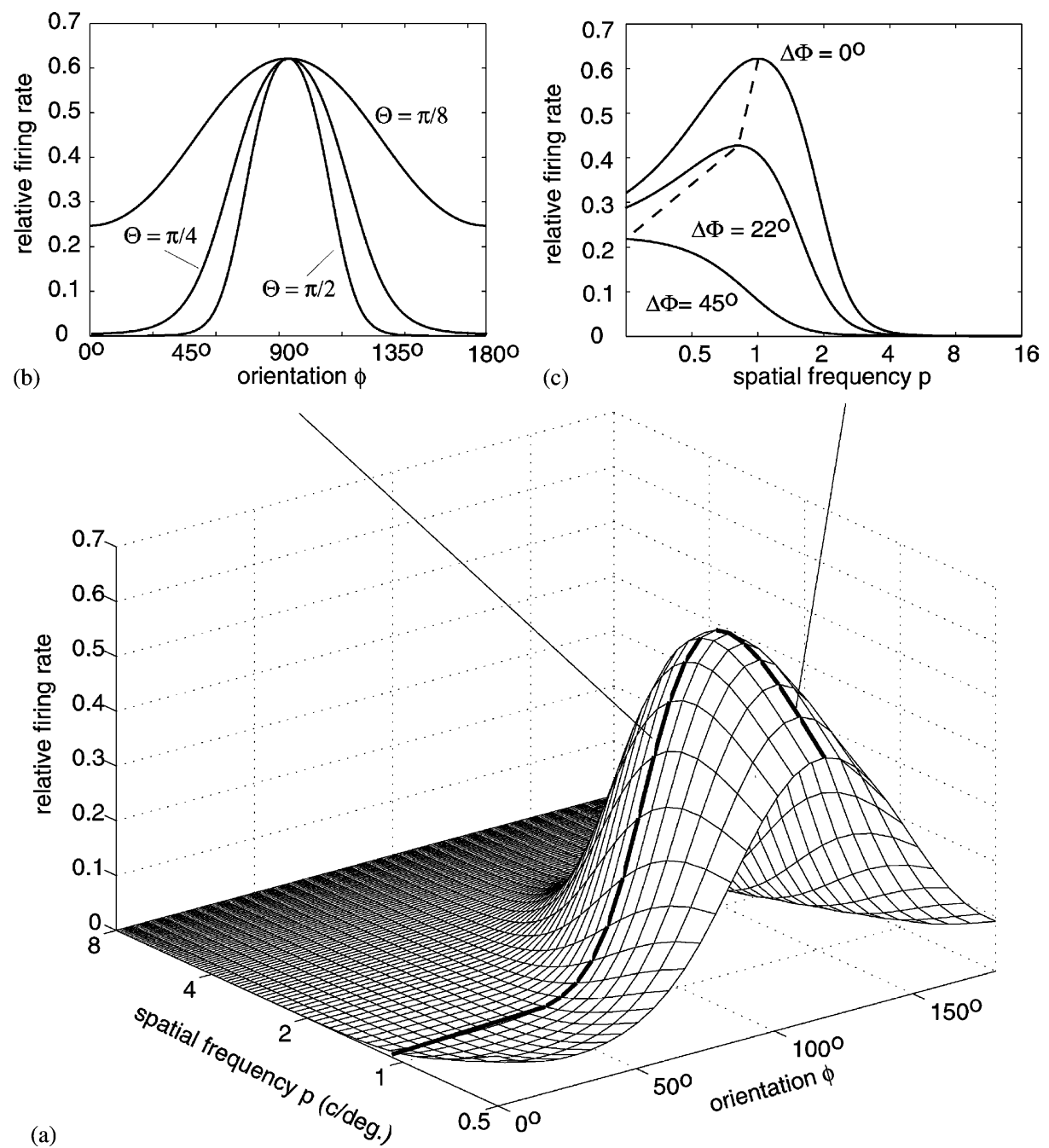

Fig. 7. Plot of normalised firing rate $\sigma(a) / \sigma_{\max }$ in response to a weakly biased stimulus from the LGN with $\Theta=\pi / 4$ and $\Phi=90^{\circ}$. Other parameters as in Fig. 6. (a) Tuning surface in the $\{p, \phi\}$-plane. (b) Orientation tuning curves for various stimulus spatial frequencies as specified by $\Theta$. The selectivity is maximal at $\Theta=\pi / 2$ (intermediate spatial frequencies). (c) Spatial frequency tuning curves for non-optimal orientations $\phi$ with $\phi=\Phi+\Delta \Phi$

\section{Lattice model of V1 and its lateral interactions}

Although the partitioning of the cortex into hypercolumns has a degree of disorder, we assume that to a first approximation each hypercolumn is a fundamental domain of a planar lattice $\mathcal{L}$, with the points of the lattice corresponding to the locations of the low frequency pinwheels. The lattice $\mathcal{L}$ is generated by two linearly independent vectors $\ell_{1}$ and $\ell_{2}$ :

$$
\mathcal{L}=\left\{m_{1} \boldsymbol{\ell}_{1}+m_{2} \boldsymbol{\ell}_{2}: m_{1}, m_{2} \in \mathbf{Z}\right\} .
$$

Let $\Psi$ be the angle between the two basis vectors $\ell_{1}$ and $\ell_{2}$. We can then distinguish three types of lattice according to the value of $\Psi$ : square lattice $(\Psi=\pi / 2)$, rhombic lattice $(0<\Psi<\pi / 2, \Psi \neq \pi / 3)$ and hexagonal $(\Psi=\pi / 3)$. 
Table 2

Generators for the planar lattices and their dual lattices

\begin{tabular}{lllll}
\hline Lattice & $L^{-1} \ell_{1}$ & $L^{-1} \ell_{2}$ & $L \hat{\ell}_{1}$ & $L \hat{\ell}_{2}$ \\
\hline Square & $(1,0)$ & $(0,1)$ & $(1,0)$ & $(0,1)$ \\
Hexagonal & $(1,0)$ & $\frac{1}{2}(-1, \sqrt{3})$ & $\left(1, \frac{1}{\sqrt{3}}\right)$ & $\left(0, \frac{2}{\sqrt{3}}\right)$ \\
Rhombic & $(1,0)$ & $(\cos \eta, \sin \eta)$ & $(1,-\cot \eta)$ & $(0, \csc \eta)$ \\
\hline
\end{tabular}

Example fundamental domains for each of these lattices are shown in Fig. 10. After rotation, the generators of the planar lattices are given in Table 2. Also shown are the generators of the dual or reciprocal lattice $\hat{\mathcal{L}}$ satisfying $\hat{\ell}_{i} \cdot \ell_{j}=\delta_{i, j}$. The lattice spacing $L=\left|\ell_{1}\right|=\left|\ell_{2}\right|$ is taken to be the average width of a hypercolumn, that is, $L \approx 1 \mathrm{~mm}$.

It follows that a local patch of (excitatory and inhibitory) cells within a hypercolumn can be uniquely labelled by the triplet $(\ell, \theta, \phi)$, where $\ell \in \mathcal{L}$ is the position of the hypercolumn on the lattice and $P:=\{\theta, \phi\}$ represents the spatial frequency and orientation preference of the cells. We assume that each isolated hypercolumn can be described by the spherical model of Section 2 and that different hypercolumns in the lattice are coupled together by long-range lateral interactions. Denoting the activity of the population $(\ell, P)$ at time time $t$ by $a(\ell, P, t)$, we introduce an evolution equation of the form

$$
\frac{\partial a(\ell, P, t)}{\partial t}=-a(\ell, P, t)+\sum_{\ell^{\prime} \in \mathcal{L}} \int w\left(\ell, P \mid \ell^{\prime}, P^{\prime}\right) \sigma\left(a\left(\ell^{\prime}, P^{\prime}, t\right)\right) D P^{\prime}+h(\ell, P, t),
$$

where $D P=\mathcal{D}(\theta, \phi)$ and

$$
w\left(\ell, P \mid \ell^{\prime}, P^{\prime}\right)=\delta_{\ell, \ell^{\prime}} W\left(P \mid P^{\prime}\right)+\beta J\left(\ell-\ell^{\prime}\right) W^{\Delta}\left(P \mid P^{\prime}\right)
$$

with $J(0)=0$. Here $W\left(P \mid P^{\prime}\right)$ denotes the $\mathbf{O}(3)$-invariant distribution of local connections within a hypercolumn, see Eq. (2.5), $W^{\Delta}\left(P \mid P^{\prime}\right)$ represents the dependence of the long-range interactions on the feature preferences of the pre- and post-synaptic cells and $J\left(\ell-\ell^{\prime}\right)$ for $\ell \neq \ell^{\prime}$ is a positive, monotonically decreasing function of cortical separation $\left|\ell-\ell^{\prime}\right|$. Thus $J(\ell)$ is invariant under discrete rotations and reflections of the lattice (see below). The coupling parameter $\beta$ determines the relative weight of the lateral connections. Microelectrode recordings suggest that $\beta$ is small and therefore that the lateral connections modulate rather than drive V1 activity [26]. Furthermore, although the lateral connections are excitatory [23,41], 20\% of the connections in superficial layers of V1 innervate inhibitory interneurons, so that the overall action of the lateral connections can become inhibitory, especially at high levels of activity [26]. It is also possible that during the experience of hallucinations there are sufficient levels of activity within V1 for the inhibitory effects of the lateral connections to predominate. In our one-population models, the sign of $\beta$ will determine whether the lateral connections have a net excitatory or inhibitory effect.

Note that we neglect all nearest neighbour interactions between hypercolumns that are mediated by local connections across hypercolumn boundaries. In order to incorporate this additional coupling into the model, it is necessary to specify in detail the spatial distribution of the orientation and spatial frequency cell preferences within each hypercolumn. Here we consider a reduced hypercolumn model (see Fig. 3), in which only a subset of neurons within a hypercolumn are explicitly represented. We assume that boundary contributions can be absorbed into the various weight functions of Eq. (3.3).

Given that the long-range horizontal connections tend to link neurons with similar feature preferences, one can construct an $\mathbf{O}$ (3)-invariant long-range distribution $W^{\Delta}$ of the form

$$
W^{\Delta}\left(P \mid P^{\prime}\right)=H\left[\cos \theta \cos \theta^{\prime}+\sin \theta \sin \theta^{\prime} \cos \left(2\left[\phi-\phi^{\prime}\right]\right)-\cos \alpha\right],
$$


where $H[x]=1$ if $x \geq 0$ and is zero otherwise. The angle $\alpha$ determines the degree of similarity in the orientation and spatial frequency preference of the linked cells and hence the patch size. It follows that the distribution (3.3) is invariant under the action of the group $\Gamma_{\mathcal{L}} \times \mathbf{O}(3)$, where $\Gamma_{\mathcal{L}}$ is the discrete symmetry group of the lattice $\mathcal{L}$. A limiting case of the above distribution is the delta function

$$
W^{\Delta}\left(P \mid P^{\prime}\right)=\frac{1}{2 \sin \theta} \delta\left(\theta-\theta^{\prime}\right) \delta\left(\phi-\phi^{\prime}\right)=\sum_{n=0}^{\infty} \sum_{m=-n}^{n} Y_{n}^{m^{*}}\left(\theta^{\prime}, \phi^{\prime}\right) Y_{n}^{m}(\theta, \phi) .
$$

However, it is likely that in two dimensions the pattern of patchy connections is more complicated than this. For example, recent optical imaging experiments combined with anatomical tracer injections suggest that the distribution of patchy horizontal connections is spatially anisotropic. Such anisotropy is particularly pronounced in the tree shrew, where differing iso-orientation patches preferentially connect to neighbouring patches in such a way as to form continuous contours following the topography of the retino-cortical map [10]. That is, the major axis of the horizontal connections tends to run parallel to the visuotopic axis of the connected cells' common orientation preference. There is also a clear anisotropy in the patchy connections of owl [44] and macaque [1] monkeys. However, in these cases most of the anisotropy can be accounted for by the fact that V1 is expanded in the direction orthogonal to ocular dominance columns. It is possible that when this expansion is factored out, there remains a weak anisotropy correlated with orientation selectivity but this remains to be confirmed experimentally. The functional role of this anisotropy has been a major focus of our recent work on the dynamics of orientation tuning in V1 $[11,17,18]$.

Anisotropy in the horizontal connections can be incorporated into the coupled lattice model (3.2) by modifying the weight distribution (3.3) along the following lines:

$$
w\left(\ell, P \mid \ell^{\prime}, P^{\prime}\right)=\delta_{\ell, \ell^{\prime}} W\left(P \mid P^{\prime}\right)+\beta J\left(\ell-\ell^{\prime}\right) W^{\Delta}\left(P \mid P^{\prime}\right) \mathcal{A}_{\ell, \ell^{\prime}}(P)
$$

with $W$ given by Eq. (3.4) or Eq. (3.5) and

$$
\mathcal{A}_{\ell, \ell^{\prime}}(P)=\frac{\chi(\theta)}{2 \eta(\theta)} H\left[\eta(\theta)-\left|\phi-\psi_{\ell, \ell^{\prime}}\right|\right],
$$

where $\psi_{\ell, \ell^{\prime}}=\arg \left(\ell-\ell^{\prime}\right)$. The parameter $\eta$ determines the degree of anisotropy, that is the angular spread of the horizontal connections around the axis joining cells with similar orientation preferences and $\chi$ is an additional normalisation factor. Both $\eta$ and $\chi$ are taken to be spatial frequency dependent as we now explain. An elegant feature of the spherical model is that it naturally incorporates the fact that, at the population level, there is zero selectivity for orientation at the pinwheels. In other words, the solution $a(\ell, \theta, \phi)$ expanded in terms of spherical harmonics is independent of $\phi$ at $\theta=0, \pi$. This implies that the lateral weight distribution (3.6) has to be isotropic at the pinwheels. In order to incorporate any anisotropy away from the pinwheels, we conclude that the spread parameter has to be $\theta$-dependent, $\eta=\eta(\theta)$ with $\eta(0)=\eta(\pi)=\pi / 2$. This is illustrated in Fig. 8 . The additional factor $\chi(\theta)$ allows for a possible correlation between the strength of the lateral connections and the spatial frequency of cells linked by these connections. There is recent data indicating that some cells located outside cytochrome oxidase (CO) blobs (regions of cells that are more metabolically active) have very little in the way of lateral connections [50], thus leading to an effective reduction in connectivity at the population level. Since the CO blobs have a strong association with the orientation singularities corresponding to low spatial frequencies $[31,35]$, the coupling may be larger around the low frequency pinwheels.

The basic rules for lateral connectivity are further illustrated in the simplified version of the model shown in Fig. 9. Here each hypercolumn is divided into cell populations characterised by one of the following set of feature preferences: (i) low spatial frequency, non-orientation selective (white discs), (ii) high spatial frequency, non-orientation selective (black discs), (iii) intermediate spatial frequency, orientation selective with preference $\phi=$ 

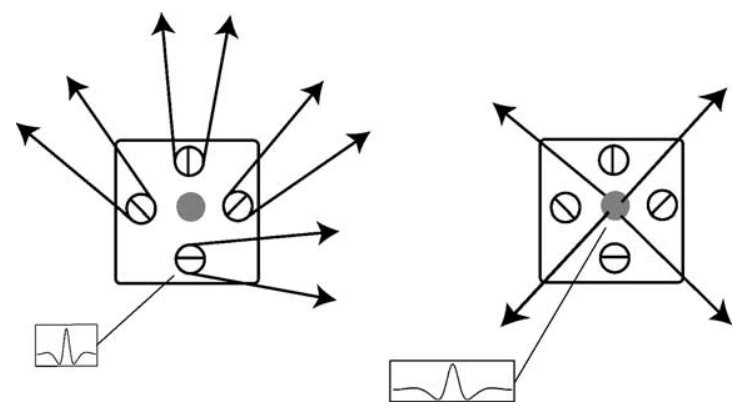

Fig. 8. Cells at intermediate spatial frequencies send out horizontal connections to cells in other hypercolumns in a direction parallel to their common preferred orientation, whereas cells at low (high) spatial frequency pinwheels connect to other low (high) pinwheels in an isotropic fashion.

$0^{\circ}, 90^{\circ}, \pm 45^{\circ}$ (white disc with oriented bar). Two examples of the resulting pattern of lateral connections between cell populations located in different hypercolumns are shown in Fig. 9. These examples are generated by selecting a particular population at each lattice location and connecting lattice points according to the following set of rules:

I. Only cells with the same spatial frequency preference are linked.

II. Cells with intermediate spatial frequency preference are only linked if they have the same orientation preference.

III. Cells with the same orientation preference are only linked if their relative cortical position is in the same direction as their common orientation preference.

An interesting mathematical property of the anisotropic weight distribution (3.6) is that it reduces the symmetry group from $\Gamma_{\mathcal{L}} \times \mathbf{O}$ (3) to $\mathbf{T}_{\mathcal{L}} \dot{+} \mathbf{D}_{n}$, where $\mathbf{T}_{\mathcal{L}}$ denotes the group of lattice translations and $\mathbf{D}_{n}, n=2,4$ or 6 , is the lattice holohedry consisting of the set of discrete rotations and reflections that preserve the lattice (see Fig. 10). The associated group action is

$$
\begin{aligned}
& \boldsymbol{\ell}_{\mathrm{s}} \cdot(\ell, \theta, \phi)=\left(\ell+\boldsymbol{\ell}_{\mathrm{s}}, \theta, \phi\right), \quad \boldsymbol{\ell}_{\mathrm{s}} \in \mathbf{T}_{\mathcal{L}}, \quad \xi \cdot(\ell, \theta, \phi)=\left(R_{\xi} \ell, \theta, \phi+\xi\right), \\
& \kappa \cdot(\ell, \theta, \phi)=\left(R_{\kappa} \ell, \theta,-\phi\right),
\end{aligned}
$$

(a)

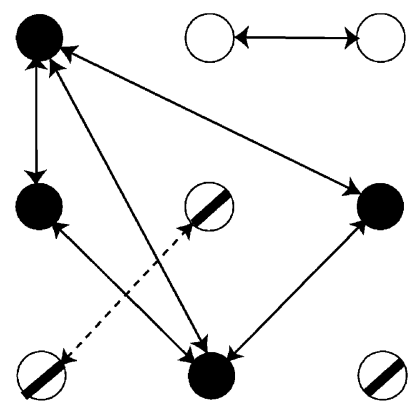

(b)

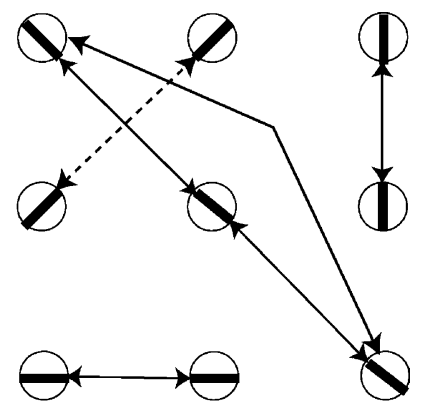

high spatial frequency, non-oriented

low spatial frequency, non-oriented

$\ominus$ intermediate spatial frequency, oriented

Fig. 9. Examples of the pattern of lateral connections between hypercolumns: (a) different spatial frequencies, (b) same spatial frequency, different orientations. See text for details. 

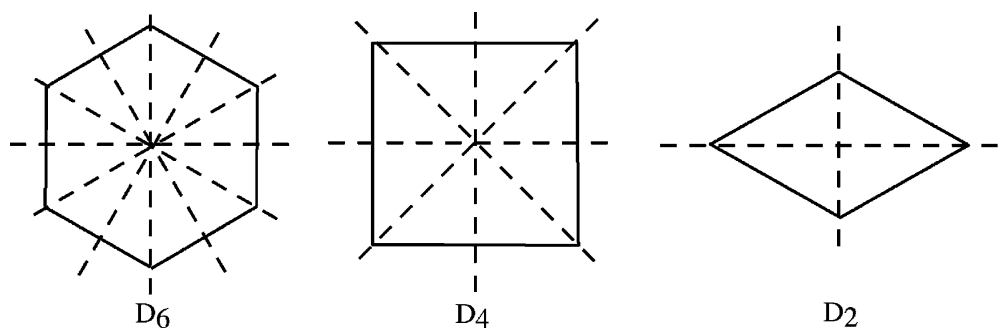

$\mathrm{D}_{2}$

Fig. 10. Holohedries of the plane.

where $(\xi, \kappa) \in D_{n}, R_{\xi}$ denotes the planar rotation through an angle $\xi$ and $R_{\kappa}$ denotes the reflection $\left(x_{1}, x_{2}\right) \mapsto$ $\left(x_{1},-x_{2}\right)$. The corresponding group action on a function $a: \mathcal{L} \times S^{2} \rightarrow \mathbf{R}$ is given by

$$
\gamma \cdot a(\ell, P)=a\left(\gamma^{-1} \cdot(\ell, P)\right) \text { for all } \gamma \in \mathbf{T}_{\mathcal{L}} \dot{+} \mathbf{D}_{n}
$$

and the invariance of $w_{\text {lat }}\left(\mathbf{r}, P \mid \mathbf{r}^{\prime}, P^{\prime}\right)$ is expressed as

$$
\gamma \cdot w\left(\ell, P \mid \ell^{\prime}, P^{\prime}\right)=w\left(\gamma^{-1} \cdot(\ell, P) \mid \gamma^{-1} \cdot\left(\ell^{\prime}, P^{\prime}\right)\right)=w\left(\ell, P \mid \ell^{\prime}, P^{\prime}\right) .
$$

It can be seen that the discrete rotation operation comprises a translation or shift of the orientation preference label $\phi$ to $\phi+\xi$, together with a rotation or twist of the position vector $\ell$ by the angle $\xi$. The spatial frequency is not affected by rotations. The fact that the weight distribution is invariant with respect to this shift-twist action has important consequences for the global dynamics of V1 in the presence of anisotropic horizontal connections (see also Ref. [11]).

\section{Cortical pattern formation}

Suppose, for concreteness, that there is a time-independent external bias $h$ such that $a(\ell, P, t)=\bar{a}$ is a fixed point solution of Eq. (3.2). Setting $a(\ell, P, t)=\bar{a}+\mathrm{e}^{\lambda t} u(\ell, P)$ and linearising about the fixed point leads to the eigenvalue equation

$$
\lambda u(\ell, P)=-u(\ell, P)+\mu \int_{0}^{\pi} \int_{0}^{\pi} \sum_{\ell^{\prime} \in \mathcal{L}} w\left(\ell, P \mid \ell^{\prime}, P^{\prime}\right) u\left(\ell^{\prime}, P^{\prime}\right) D P^{\prime}
$$

with $P=(\theta, \phi), P^{\prime}=\left(\theta^{\prime}, \phi^{\prime}\right)$ and $D P^{\prime}=\sin \theta^{\prime} \mathrm{d} \theta^{\prime} \mathrm{d} \phi^{\prime} / 2 \pi$. Since the weight distribution $w$ is bounded, it follows that when the network is in a low activity state $\bar{a}$ such that $\mu=\sigma^{\prime}(\bar{a}) \approx 0$, any solution of Eq. (4.1) satisfies $\operatorname{Re} \lambda<0$ and the fixed point is linearly stable. However, when the excitability of the network is increased, either through the action of some hallucinogen or through external stimulation, $\mu$ increases. This can induce a Turing instability [47], leading to the formation of spontaneous cortical activity patterns. In this section, we derive conditions for the onset of a Turing instability and discuss the selection and stability of the patterns using weakly nonlinear analysis.

\subsection{Isotropic and homogeneous lateral connections}

Consider first the case of isotropic and homogeneous long-range connections. Substitution of Eq. (3.3) into Eq. (4.1) gives

$$
\left[\frac{\lambda+1}{\mu}\right] u(\ell, P)=\int_{0}^{\pi} \int_{0}^{\pi} W\left(P \mid P^{\prime}\right) u\left(\ell, P^{\prime}\right) D P^{\prime}+\beta \sum_{\ell^{\prime} \neq \ell} J\left(\ell-\ell^{\prime}\right) \int_{0}^{\pi} \int_{0}^{\pi} W^{\Delta}\left(P \mid P^{\prime}\right) u\left(\ell^{\prime}, P^{\prime}\right) D P^{\prime} .
$$



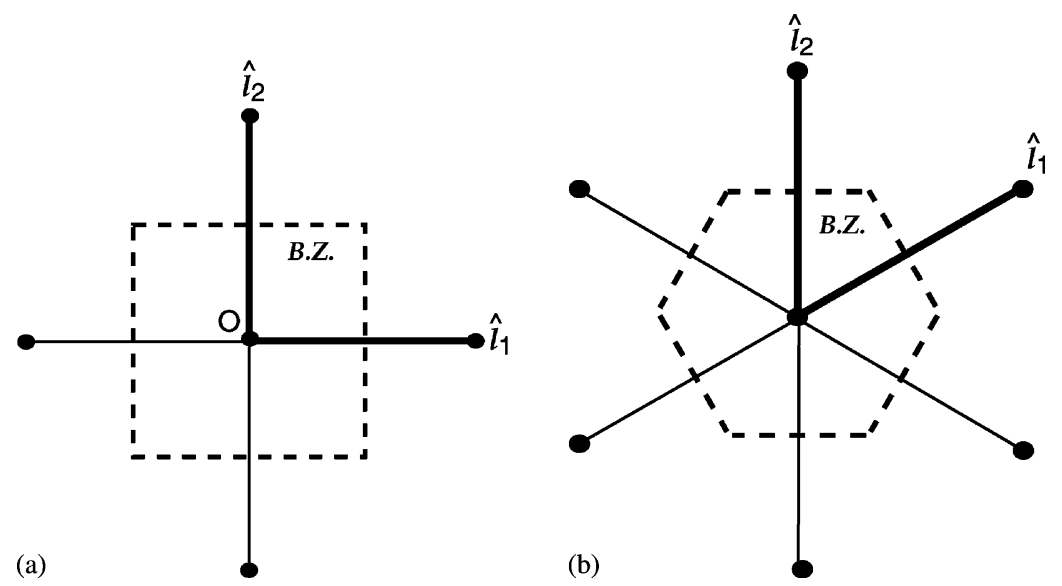

Fig. 11. Construction of the first Brillouin zone in (a) the reciprocal square lattice, (b) the reciprocal hexagonal lattice.

Since the weight distribution $w$ is invariant under the action of $\Gamma_{\mathcal{L}} \times \mathbf{O}(3)$, it follows that the eigensolutions are of the form

$$
u(\ell, P)=\mathrm{e}^{\mathrm{ik} \cdot \ell} Y_{n}^{m}(\theta, \phi)
$$

for $n \in \mathbf{Z},-n \leq m \leq n$ and $\mathbf{k}=k_{1} \hat{\ell}_{1}+k_{2} \hat{\ell}_{2}$, where $\hat{\ell}_{j}$ are the generators of the reciprocal lattice $\hat{\mathcal{L}}$. We restrict $\mathbf{k}$ to lie in the first Brillouin zone $\mathcal{U}$ of the reciprocal lattice $\hat{\mathcal{L}}$ [3]. The first Brillouin zone is the fundamental domain around the origin of the reciprocal lattice formed by the perpendicular bisectors of the shortest lattice vectors (of length $2 \pi / L$ ). Examples for the square and hexagonal lattices are shown in Fig. 11. We also assume periodic boundary conditions for the activity

$$
u\left(\ell+N \ell_{1}, P\right)=u\left(\ell+N \ell_{2}, P\right)=u(\ell, P)
$$

for some integer $N \gg 1$ so that the wavevectors are discretised, $k_{i}=2 \pi n_{i} / N$ for integers $n_{i}$. The corresponding eigenvalue $\lambda=\lambda_{n}(\mathbf{k})$ is $(2 n+1)$-fold degenerate such that

$$
\lambda_{n}(\mathbf{k})=-1+\mu\left[\tilde{W}_{n}+\beta \tilde{J}(\mathbf{k}) \tilde{W}_{n}^{\Delta}\right],
$$

where $\tilde{W}_{n}$ and $\tilde{W}_{n}^{\Delta}$ are the $n$th coefficients in the spherical harmonic expansions of $W$ and $W^{\Delta}$, see Eq. (2.9) and

$$
\tilde{J}(\mathbf{k})=\sum_{\ell \in \mathcal{L}} J(\ell) \mathrm{e}^{-\mathrm{ik} \cdot \ell}
$$

In the case $\beta=0$, each isolated hypercolumn is described by the spherical model (2.2) and the eigenvalues $\lambda_{n}$ are k-independent. Assuming that $\tilde{W}_{1}>\tilde{W}_{n}$ for all $n \neq 1$, the condition for marginal stability reduces to $\mu_{\mathrm{c}}=1 / \tilde{W}_{1}$ such that there exists an $\mathbf{O}(3)$-invariant submanifold of marginally stable states involving linear combinations of the three first order spherical harmonics:

$$
u(\ell, \theta, \phi)=C[\cos \theta \cos \bar{\theta}(\ell)+\sin \theta \sin \bar{\theta}(\ell) \cos (2[\phi-\bar{\phi}(\ell)])]
$$

for arbitrary phases $\bar{\theta}(\ell), \bar{\phi}(\ell)$. Thus the hypercolumn at $\ell \in \mathcal{L}$ exhibits a tuning surface of the form shown in Fig. 6 , with the peak located at $(\bar{\theta}(\ell), \bar{\phi}(\ell))$.

If the lateral connections are now switched on, there is a k-dependent splitting of the degenerate eigenvalue $\lambda_{1}$. Since the long-range connections are narrowly tuned with respect to orientation and spatial frequency, the 
corresponding spherical harmonic coefficients $\tilde{W}_{n}$ are only weakly dependent on $n \in \mathbf{Z}$. This implies that the horizontal connections do not excite other spherical harmonic components within a hypercolumn and the condition for marginal stability of the homogeneous fixed point is obtained from the eigenvalue equation

$$
\frac{\lambda_{1}(\mathbf{k})+1}{\mu}=\frac{1}{\mu_{\mathrm{c}}}+\beta \tilde{J}(\mathbf{k})
$$

where a positive factor $\tilde{W}_{1}^{\Delta}$ has been absorbed into $\beta$. Suppose that $\beta \tilde{J}(\mathbf{k})$ has a global positive maximum at $\mathbf{k}=\mathbf{k}_{\mathrm{c}} \neq 0$. The homogeneous fixed point is then marginally stable at the critical value

$$
\hat{\mu}_{\mathrm{c}}=\frac{\mu_{\mathrm{c}}}{1+\beta \mu_{\mathrm{c}} \tilde{J}\left(\mathbf{k}_{\mathrm{c}}\right)} .
$$

Since $\tilde{J}(\mathbf{k})$ is invariant with respect to the corresponding lattice holohedry $\mathbf{D}_{n}$, all other wavevectors related to $\mathbf{k}_{\mathrm{c}}$ by a discrete rotation will also be selected. The marginally stable eigenmodes will thus be of the form

$$
u(\ell, \theta, \phi)=C(\ell)[\cos \theta \cos \bar{\theta}+\sin \theta \sin \bar{\theta} \cos (2[\phi-\bar{\phi}])]
$$

for arbitrary constant phases $\bar{\theta}, \bar{\phi}$ and

$$
C(\ell)=\sum_{j=1, \ldots, N}\left[u_{j} \mathrm{e}^{\mathrm{i} \mathbf{k}_{j} \cdot \ell}+u_{j}^{*} \mathrm{e}^{-\mathbf{i} \mathbf{k}_{j} \cdot \ell}\right]
$$

where $u_{j}$ is a complex amplitude with conjugate $u_{j}^{*}$. Here $N=2$ for the square lattice with $\mathbf{k}_{1}=\mathbf{k}_{\mathrm{c}}$ and $\mathbf{k}_{2}=$ $R_{\pi / 2} \mathbf{k}_{\mathrm{c}}$, where $R_{\xi}$ denotes rotation through an angle $\xi$. Similarly, $N=3$ for the hexagonal lattice with $\mathbf{k}_{1}=\mathbf{k}_{\mathrm{c}}$, $\mathbf{k}_{2}=R_{2 \pi / 3} \mathbf{k}_{\mathrm{c}}$ and $\mathbf{k}_{3}=R_{4 \pi / 3} \mathbf{k}_{\mathrm{c}}=-\mathbf{k}_{1}-\mathbf{k}_{2}$.

The pattern generated by the eigensolution (4.10) with $\mathbf{k}_{\mathrm{c}} \neq 0$ consists of a distribution of tuning surfaces across cortex whose peak response alternates between the points $(\bar{\theta}, \bar{\phi})$ and $(\pi-\bar{\theta}, \bar{\phi}+\pi / 2)$ according to the sign of the amplitude $C(\ell)$. For wavevectors $\mathbf{k}_{\mathrm{c}}$ that are commensurate with the lattice such alternations in sign generate a periodic tiling of the cortical plane consisting of stripes, hexagons or squares, whereas incommensurate wavevectors generate quasi-periodic patterns. It is important to emphasise that doubly periodic solutions arise naturally in this model due to the presence of a lattice of orientation pinwheels around which the hypercolumns are organised. In previous continuum models of cortical pattern formation [11,21], double periodicity was imposed by hand as a mathematical simplification rather than as a consequence of a physical lattice.

As an illustrative example, consider a long-range distance function for the square lattice that satisfies

$$
J\left(n_{1} \ell_{1}+n_{2} \ell_{2}\right)=\delta_{n_{1}, \pm 1} \delta_{n_{2}, 0}+\delta_{n_{1}, 0} \delta_{n_{2}, \pm 1}+A_{1} \delta_{n_{1}, \pm 1} \delta_{n_{2}, \pm 1}+A_{2}\left[\delta_{n_{1}, \pm 2} \delta_{n_{2}, 0}+\delta_{n_{1}, 0} \delta_{n_{2}, \pm 2}\right]
$$

with $A_{2}<A_{1}<1$. The corresponding Fourier transform is

$$
\tilde{J}(\mathbf{k})=2\left(\cos k_{x}+\cos k_{y}+A_{1}\left[\cos \left(k_{x}+k_{y}\right)+\cos \left(k_{x}-k_{y}\right)\right]+A_{2}\left[\cos 2 k_{x}+\cos 2 k_{y}\right]\right)
$$

for $\mathbf{k}=\left(k_{x}, k_{y}\right)$. Contour plots of $\tilde{J}(\mathbf{k})$ as a function of $\mathbf{k}$ are shown in Fig. 12 with $\mathbf{k}$ restricted to lie in the first Brillouin zone. One can see that there is a global maximum at $\mathbf{k}=0$ and four global minima, reflecting the fourfold symmetry of the lattice. (Also shown is the corresponding contour plot for nearest neighbour coupling on an hexagonal lattice where there is a sixfold symmetry.) This means that in the excitatory regime $(\beta>0)$, there is a bulk instability with respect to the lattice, but a Turing instability with respect to orientation and spatial frequency. Thus each hypercolumn exhibits a tuning surface with the same peak response. On the other hand, in the inhibitory regime $(\beta<0)$ the marginally stable modes have a critical wavenumber $\mathbf{k}_{\mathrm{c}} \neq 0$, implying that there is a Turing instability with respect to both the internal and external degrees of freedom.

The above example establishes that a Turing instability on the lattice can be induced by inhibitory lateral interactions (without excitation) since there is a gap in the interactions at the origin, that is, $J(0)=0$. This differs from 

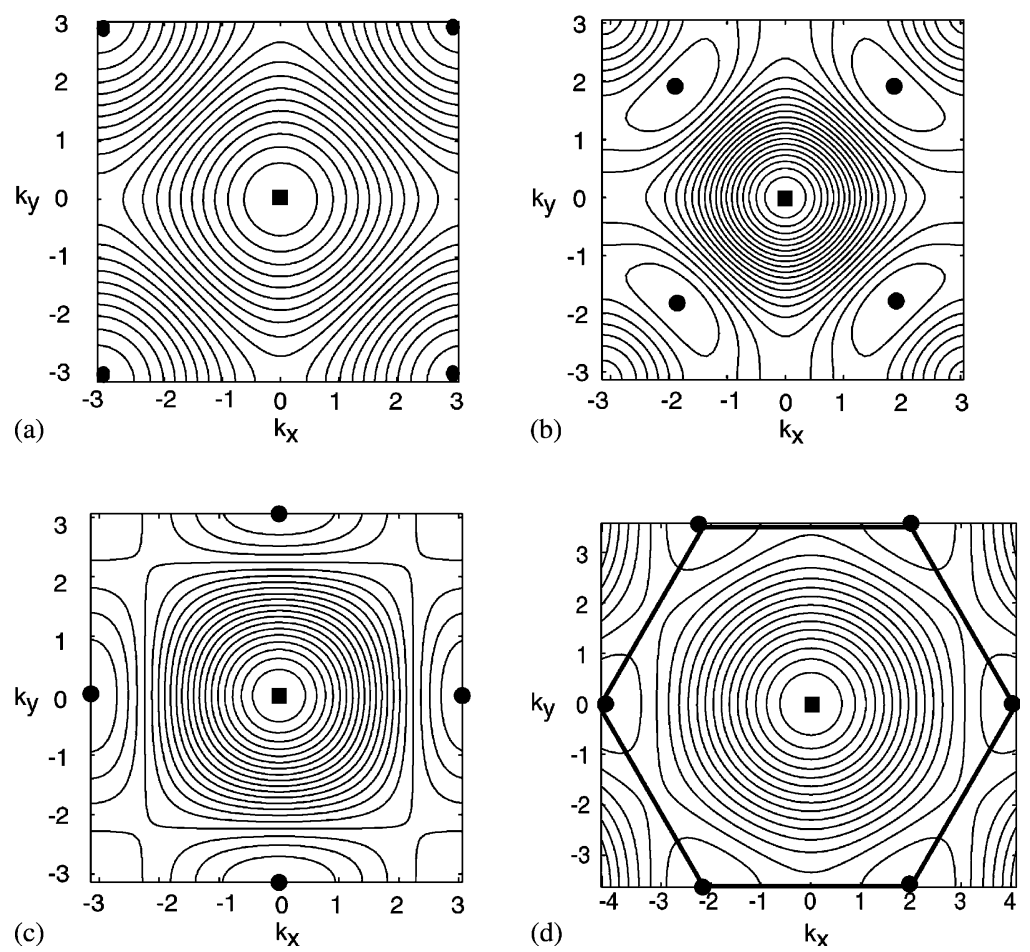

Fig. 12. (a-c) Contour plot of $\tilde{J}(\mathbf{k})$ satisfying Eq. (4.13) for $\mathbf{k}=\left(k_{x}, k_{y}\right)$ in the first Brillouin zone of the square lattice. Filled circles indicate locations of global minima, whereas filled square indicates location of global maximum at origin. (a) $A_{1}=A_{2}=0$ with four minima at $\left(k_{x}, k_{y}\right)=( \pm \pi, \pm \pi)$. (b) $A_{1}=0.8$ and 0.6 with four minima inside the Brillouin zone boundary. (c) $A_{1}=0.8, A_{2}=0$ with four minima at $\left(k_{x}, k_{y}\right)=( \pm \pi, 0)$ and $\left(k_{x}, k_{y}\right)=(0, \pm \pi)$. (d) Contour plot of $\tilde{J}(\mathbf{k})$ for nearest neighbour coupling on the hexagonal lattice showing a sixfold symmetry.

the standard mechanism for neural pattern formation based on a combination of excitation and inhibition, which is the mechanism by which the local recurrent circuitry generates orientation and spatial frequency tuning within a hypercolumn (see Section 3). To reinforce this idea, we consider the example of a Gaussian distance function $J(\ell)=\mathrm{e}^{-\ell^{2} / 2 \sigma^{2}}$ on a square lattice for which

$$
\tilde{J}(\mathbf{k})=\left[2 \sum_{n_{1} \geq 0} \mathrm{e}^{-L^{2} n_{1}^{2} / 2 \sigma^{2}} \mathrm{e}^{\mathrm{i} k_{x} n_{1}}-2\right]\left[2 \sum_{n_{2} \geq 0} \mathrm{e}^{-L^{2} n_{2}^{2} / 2 \sigma^{2}} \mathrm{e}^{\mathrm{i} k_{y} n_{2}}-2\right],
$$

where $\mathbf{k}=k_{x} \hat{\ell}_{1}+k_{y} \hat{\ell}_{2}$ and $\boldsymbol{\ell}=n_{1} \ell_{1}+n_{2} \ell_{2}$. Suppose that we can approximate the sums by Gaussian integrals (which is a good approximation when $\sigma \gg L$ ), so that

$$
\tilde{J}(\mathbf{k}) \approx\left[\frac{\sqrt{\pi} \sigma}{L} \mathrm{e}^{-\sigma^{2} k_{x}^{2} / 2 L^{2}}-2\right]\left[\frac{\sqrt{\pi} \sigma}{L} \mathrm{e}^{-\sigma^{2} k_{y}^{2} / 2 L^{2}}-2\right] .
$$

The resulting function is plotted in Fig. 13 for $L=0.15 \sigma$. It can be seen that there is a global positive maximum at $\mathbf{k}=0$ and four global negative minima at $\mathbf{k}=(0, \pm \pi),( \pm \pi, 0)$. As in the previous example, a Turing instability occurs for inhibitory lateral connections due to the existence of a gap in the Gaussian at the origin. 


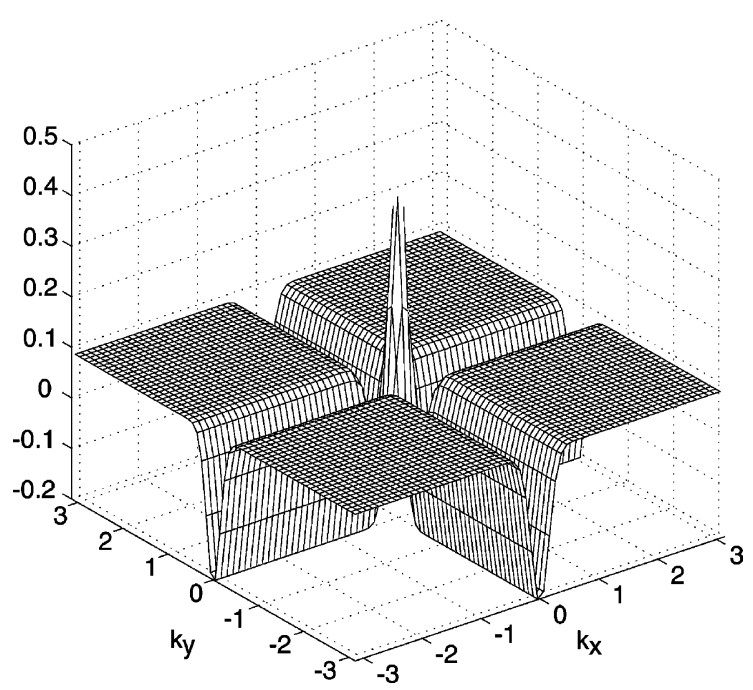

Fig. 13. Surface plot of $\tilde{J}(\mathbf{k})$ (in arbitrary units) with $\mathbf{k}=\left(k_{x}, k_{y}\right)$ for a Gaussian distance function with $L=0.15 \sigma$.

\subsection{Anisotropic lateral connections}

Suppose that we now substitute the anisotropic weight distribution (3.6) into Eq. (4.1) with $W^{\Delta}$ given by the delta function (3.5). Since the weights are invariant with respect to discrete translations in the lattice, it follows that the eigensolutions have the general form $u(\ell, P)=\mathrm{e}^{\mathrm{i} \mathbf{k} \cdot \ell} u_{\mathbf{k}}(P)$ such that

$$
\left[\frac{\lambda+1}{\mu}\right] u_{\mathbf{k}}(P)=\int W\left(P \mid P^{\prime}\right) u_{\mathbf{k}}\left(P^{\prime}\right) D P^{\prime}+\beta \tilde{J}(\mathbf{k} \mid P) u_{\mathbf{k}}(P) .
$$

Here $\tilde{J}(\mathbf{k} \mid P)$ is defined according to

$$
\tilde{J}(\mathbf{k} \mid P)=\sum_{\ell^{\prime} \neq \ell} J\left(\ell-\ell^{\prime}\right) \mathcal{A}_{\ell, \ell^{\prime}}(P) \mathrm{e}^{\mathrm{ik} \cdot\left(\ell^{\prime}-\ell\right)}
$$

which can be rewritten as

$$
\tilde{J}(\mathbf{k} \mid P)=\frac{\chi}{2 \eta} \sum_{\ell \neq 0} H\left(\eta-\left|\phi-\psi_{\ell}\right|\right) J(\ell) \mathrm{e}^{\mathrm{i} k|\ell| \cos \left(\psi_{\ell}-\varphi\right)},
$$

where $\mathbf{k}=k(\cos \varphi, \sin \varphi)$ and $\ell=|\ell|\left(\cos \psi_{\ell}, \sin \psi_{\ell}\right)$. Recall that the degree of anisotropy $\eta$ and the coupling $\chi$ are both generally $\theta$-dependent. Expanding $u_{\mathbf{k}}(P)$ in terms of spherical harmonics

$$
u_{\mathbf{k}}(P)=\sum_{n \in \mathbf{Z}} \sum_{m=-n}^{n} U_{n m}(\mathbf{k}) Y_{n}^{m}(\theta, \phi)
$$

leads to the matrix eigenvalue equation

$$
\left[\frac{\lambda+1}{\mu}-\tilde{W}_{n}\right] U_{n m}(\mathbf{k})=\beta \sum_{n^{\prime} \in \mathbf{Z}} \sum_{m^{\prime}=-n^{\prime}}^{n^{\prime}} \tilde{J}_{n m, n^{\prime} m^{\prime}}(\mathbf{k}) U_{n^{\prime} m^{\prime}}(\mathbf{k})
$$


with

$$
\tilde{J}_{n m, n^{\prime} m^{\prime}}(\mathbf{k})=2 \int_{0}^{\pi} \int_{0}^{\pi} Y_{n}^{m^{*}}(\theta, \phi) Y_{n^{\prime}}^{m^{\prime}}(\theta, \phi) \tilde{J}(\mathbf{k} \mid \theta, \phi) \sin \theta \mathrm{d} \theta \mathrm{d} \phi .
$$

In order to solve the matrix equation (4.20), we exploit the experimental observation that the lateral connections are weak relative to the local connections and carry out a perturbation expansion in the small parameter $\beta$. As in our analysis of the isotropic case, we assume $\tilde{W}_{1}=\max \left\{\tilde{W}_{n}, n \in \mathbf{Z}^{+}\right\}>0$ so that in the limit $\beta \rightarrow 0$, the homogeneous state becomes marginally stable at the critical point $\mu_{\mathrm{c}}=1 / \tilde{W}_{1}$ due to excitation of the eigenmodes (4.7). Each hypercolumn has a threefold degeneracy and there are no $\ell$-dependent correlations between tuning surfaces. On the other hand, when $\beta>0$ there is a $\mathbf{k}$-dependent splitting of the eigenvalue $\lambda_{1}=-1+\mu \tilde{W}_{1}$ that also separates out the first order spherical harmonics (since the anisotropic weights explicitly break $\mathbf{O}(3)$ symmetry). Denoting the characteristic size of such a splitting by $\delta \lambda=\mathcal{O}(\beta)$, we impose the condition that $\delta \lambda \ll \mu \Delta \tilde{W}$, where $\Delta \tilde{W}=\min \left\{\tilde{W}_{1}-\tilde{W}_{m}, m \neq 1\right\}$. This ensures that the perturbation does not excite states associated with other eigenvalues of the unperturbed problem and we can then restrict ourselves to calculating perturbative corrections to $\lambda_{1}$. Thus, introduce the perturbation expansions

$$
\begin{aligned}
& \lambda=-1+\mu \tilde{W}_{1}+\beta \lambda^{(1)}+\beta^{2} \lambda^{(2)}+\cdots, \\
& U_{n m}=U_{m} \delta_{n, 1}+\beta U_{n m}^{(1)}+\beta^{2} U_{n m}^{(2)}+\cdots
\end{aligned}
$$

and substitute these into the eigenvalue equation (4.20). We then systematically solve the resulting hierarchy of equations to successive orders in $\beta$, taking into account the fact that the unperturbed problem is degenerate. Here we will only describe the lowest order corrections, obtained by setting $n=1 \mathrm{in}$ Eq. (4.20) to yield the $\mathbf{O}(\beta)$ eigenvalue equation

$$
\sum_{m^{\prime}=-1}^{1} \tilde{J}_{m m^{\prime}}(\mathbf{k}) U_{m^{\prime}}=\lambda^{(1)} U_{m},
$$

where $\tilde{J}_{m m^{\prime}}(\mathbf{k})=\tilde{J}_{1 m 1 m^{\prime}}(\mathbf{k})$.

Evaluating Eqs. (4.18) and (4.21) for $n=n^{\prime}=1$, we obtain the matrix equation

$$
\left(\begin{array}{ccc}
b G(\mathbf{k}) & b_{1} G_{1}(\mathbf{k}) & b_{1} G_{-1}(\mathbf{k}) \\
b_{1} G_{-1}(\mathbf{k}) & b_{0} G(\mathbf{k}) & b_{2} G_{-2}(\mathbf{k}) \\
b_{1} G_{1}(\mathbf{k}) & b_{2} G_{2}(\mathbf{k}) & b_{0} G(\mathbf{k})
\end{array}\right)\left(\begin{array}{c}
U_{0} \\
U_{1} \\
U_{-1}
\end{array}\right)=\lambda^{(1)}(\mathbf{k})\left(\begin{array}{c}
U_{0} \\
U_{1} \\
U_{-1}
\end{array}\right),
$$

where

$$
\begin{aligned}
& G(\mathbf{k})=\sum_{\ell} \cos \left(k|\ell| \cos \left(\psi_{\ell}-\varphi\right)\right) J(\ell) \\
& G_{ \pm r}(\mathbf{k})=\sum_{\ell} \exp \left( \pm 2 \mathrm{i} r \psi_{\ell}\right) \cos \left(k|\ell| \cos \left(\psi_{\ell}-\varphi\right)\right) J(\ell), \quad r=1,2
\end{aligned}
$$

and

$$
\begin{aligned}
& b=\frac{3}{4 \pi} \int_{0}^{\pi} \chi(\theta) \cos ^{2} \theta \sin \theta \mathrm{d} \theta, \\
& b_{0}=\frac{3}{8 \pi} \int_{0}^{\pi} \chi(\theta) \sin ^{3} \theta \mathrm{d} \theta,
\end{aligned}
$$




$$
\begin{aligned}
& b_{1}=\frac{3 \sqrt{2}}{8 \pi} \int_{0}^{\pi} \frac{\sin [2 \eta(\theta)]}{2 \eta(\theta)} \chi(\theta) \cos \theta \sin ^{2} \theta \mathrm{d} \theta, \\
& b_{2}=\frac{3}{8 \pi} \int_{0}^{\pi} \frac{\sin [4 \eta(\theta)]}{4 \eta(\theta)} \chi(\theta) \sin ^{3} \theta \mathrm{d} \theta .
\end{aligned}
$$

It follows, to first order in $\beta$, that there are three dispersion branches $\lambda_{s}(\mathbf{k}), s=0, \pm$ :

$$
\lambda_{S}(\mathbf{k})=-1+\mu\left[\tilde{W}_{1}+\beta \lambda_{s}^{(1)}(\mathbf{k})\right],
$$

where $\lambda_{s}(\mathbf{k})$ are the eigenvalues of the matrix on the left-hand side of Eq. (4.25). Let $\beta<0$ and suppose that $\lambda_{s}^{(1)}(\mathbf{k})$ has a global minimum at $\mathbf{k}_{s}$ for $s=0, \pm$. If $\Lambda\left(\mathbf{k}_{\mathrm{c}}\right)=\min \left\{\lambda_{s}^{(1)}\left(\mathbf{k}_{s}\right), s=0, \pm\right\}$ then a Turing instability on the lattice occurs at the critical point

$$
\hat{\mu}_{\mathrm{c}}=\frac{1}{\tilde{W}_{1}+\beta \Lambda\left(\mathbf{k}_{\mathrm{c}}\right)} .
$$

The corresponding marginally stable eigenmodes are of the general form

$$
u(\ell, \theta, \phi)=\sum_{j=1}^{N} u_{j} \mathrm{e}^{\mathrm{i} \mathbf{k}_{j} \cdot \ell}\left[\sum_{m=0, \pm 1} Y_{1}^{m}(\theta, \phi) U_{m}\left(\mathbf{k}_{j}\right)\right]+\text { c.c. }
$$

where $\mathbf{U}\left(\mathbf{k}_{j}\right)$ is the eigenvector associated with the minimal eigenvalue $\Lambda\left(\mathbf{k}_{j}\right)$ and the wavevectors are as defined below Eq. (4.11). We use the shorthand notation c.c. to denote complex conjugate. Thus the degeneracy with respect to the three first order spherical harmonics is explicitly broken. However, recall from Section 3 that the anisotropic connections are invariant with respect to a shift-twist lattice symmetry. This implies that

$$
U_{m}\left(R_{\xi} \mathbf{k}\right)=c \mathrm{e}^{-2 \mathrm{i} m \xi} U_{m}(\mathbf{k})
$$

for some constant $c$. Full $\mathbf{O}(3)$ symmetry is recovered when the lateral connections are isotropic and homogeneous, since $b_{1}=b_{2}=0$ and $b_{0}=b$ so that the three dispersion branches merge.

\subsubsection{Homogeneous case}

Further simplification occurs if the strengths or weights of the lateral interactions are independent of spatial frequency so that $\chi(\theta)=\chi_{0}$ for all $\theta \in[0, \pi]$. Choosing the normalisation $\chi_{0}=2 \pi$, we find that $b=b_{0}=1$ and $\left|b_{2}\right|<1$. Moreover, since $\eta(\theta)$ is symmetric under $\theta \rightarrow \pi-\theta$, it follows that $b_{1}=0$. Hence, the eigenvalue solutions of Eq. (4.25) reduce to

$$
\lambda_{-}^{(1)}(\mathbf{k})=G(\mathbf{k})-b_{2}\left|G_{2}(\mathbf{k})\right|
$$

with eigenmode $\mathbf{U}_{-}(\mathbf{k})=(0,1,-F(\mathbf{k}))$

$$
\lambda_{+}^{(1)}(\mathbf{k})=G(\mathbf{k})+b_{2}\left|G_{2}(\mathbf{k})\right|
$$

with eigenmode $\mathbf{U}_{+}(\mathbf{k})=(0,1, F(\mathbf{k}))$ and

$$
\lambda_{0}^{(1)}(\mathbf{k})=G(\mathbf{k})
$$

with eigenmode $\mathbf{U}_{0}=(1,0,0)$. We have defined $F(\mathbf{k})=G_{2}(\mathbf{k}) /\left|G_{2}(\mathbf{k})\right|$. Since $\left|b_{2}\right|<1$, it follows that either the even mode $\mathbf{U}_{+}$or the odd mode $\mathbf{U}_{-}$becomes marginally stable first; which of these two types of mode is selected depends on the sign of $b_{2}$. 
It is easy to establish that the sign of $b_{2}$ depends on the degree of anisotropy. For example, if $\eta(\theta)=\eta_{0}$ except in small regions around the pinwheels then

$$
b_{2} \approx \frac{\sin 4 \eta_{0}}{4 \eta_{0}}
$$

Under such an approximation, $b_{2}>0$ when $\eta_{0}<\pi / 4$, which corresponds to strong anisotropy in the linear zones (away from the pinwheels). In this case,

$$
\min _{\mathbf{k}}\left\{\lambda_{-}^{(1)}(\mathbf{k})\right\}<\min _{\mathbf{k}}\left\{\lambda_{0}^{(1)}(\mathbf{k})\right\}<\min _{\mathbf{k}}\left\{\lambda_{+}^{(1)}(\mathbf{k})\right\}
$$

Let $\mathbf{k}_{\mathrm{c}}$ be a critical wavevector and set $4 \phi_{\mathrm{c}}=\arg F\left(\mathbf{k}_{\mathrm{c}}\right)$. Using the fact that $F\left(R_{\psi} \mathbf{k}\right)=\mathrm{e}^{4 \mathrm{i} \psi} F(\mathbf{k})$, see Eq. (4.27), it follows that the marginally stable modes are of the form

$$
u_{-}(\boldsymbol{\ell}, \theta, \phi)=\left[\sum_{j=1}^{N} u_{j} \sin \left(2\left[\phi-\phi_{\mathrm{c}}-\psi_{j}\right]\right) \mathrm{e}^{\mathrm{i} \mathbf{k}_{j} \cdot \boldsymbol{\ell}}+\text { c.c. }\right] \sin \theta
$$

with $\mathbf{k}_{j}=R_{\psi_{j}} \mathbf{k}_{\mathrm{c}}$ and $\psi_{1}=0$. The factor $\sin \theta$ implies that each hypercolumn has a tuning surface that peaks at intermediate frequencies $(\theta=\pi / 2)$. When there is weaker anisotropy within the linear zones such that $b_{2}<0$, we have

$$
\min _{\mathbf{k}}\left\{\lambda_{+}^{(1)}(\mathbf{k})\right\}<\min _{\mathbf{k}}\left\{\lambda_{0}^{(1)}(\mathbf{k})\right\}<\min _{\mathbf{k}}\left\{\lambda_{-}^{(1)}(\mathbf{k})\right\}
$$

and the marginally stables modes are of the form

$$
u_{+}(\boldsymbol{\ell}, \theta, \phi)=\left[\sum_{j=1}^{N} u_{j} \cos \left(2\left[\phi-\phi_{\mathrm{c}}-\psi_{j}\right]\right) \mathrm{e}^{\mathrm{i} \mathbf{k}_{j} \cdot \boldsymbol{\ell}}+\text { c.c. }\right] \sin \theta
$$

\subsubsection{Nearest neighbour coupling}

As a specific example, consider nearest neighbour coupling with $J(\ell)=1$ if $\boldsymbol{\ell}=\left(L \cos \psi_{j}, L \sin \psi_{j}\right), j=$ $1, \ldots, N$ and zero otherwise. Here we are expressing lattice points in Cartesian coordinates such that $\psi_{j}=$ $2 \pi(j-1) / N$ with $N=4$ for a square lattice and $N=6$ for a hexagonal lattice. (For a rhomboid lattice, we would have $\psi_{j}=0, \psi, \pi, \pi+\psi$ with $0<\psi<\pi / 2, \psi \neq \pi / 3$.) In the case of a square lattice, Eqs. (4.26) and (4.27) simplify to

$$
\begin{aligned}
& G(\mathbf{k})=2\left[\cos \left(k_{x}\right)+\cos \left(k_{y}\right)\right], \\
& G_{ \pm r}(\mathbf{k})=2\left[\cos \left(k_{x}\right)+(-1)^{r} \cos \left(k_{y}\right)\right], \quad r=1,2 .
\end{aligned}
$$

The critical wavevectors are $\mathbf{k}_{j}=( \pm \pi, \pm \pi)$ with $F\left(\mathbf{k}_{\mathrm{c}}\right)=-1$ and $\phi_{\mathrm{c}}=\pi / 4$. Hence the odd/even modes are given by

$$
u_{-}(\ell, \theta, \phi)=C(\ell) \cos 2 \phi \sin \theta, \quad u_{+}(\ell, \theta, \phi)=C(\ell) \sin 2 \phi \sin \theta .
$$

In Fig. 14, we show examples of even and odd roll patterns corresponding to a plane wave with critical wavevector $\mathbf{k}_{\mathrm{c}}=(\pi,-\pi)$. These are constructed using a winner-take-all rule in which only the orientation of maximal response within each hypercolumn on the lattice is shown. (For this particular example, combining orthogonal rolls of the same type does not introduce a new pattern.) 


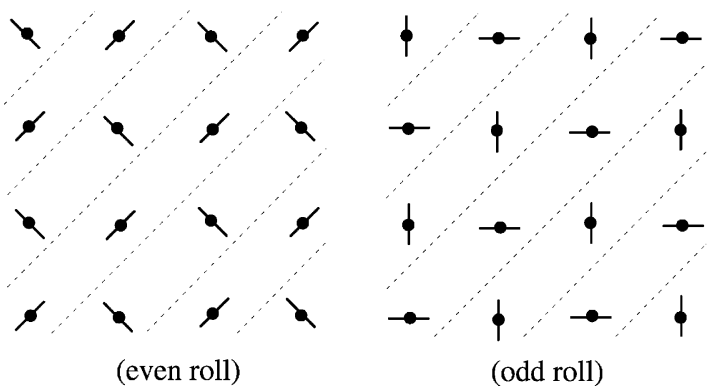

Fig. 14. Even and odd roll patterns on a square lattice with nearest neighbour lateral coupling. Dashed lines highlight the direction of the rolls.

In the case of a hexagonal lattice, Eqs. (4.26) and (4.27) become

$$
\begin{aligned}
& G(\mathbf{k})=2\left[\cos \left(k_{x}\right)+\cos \left(\frac{1}{2} k_{x}+\frac{\sqrt{3}}{2} k_{y}\right) \cos \left(-\frac{1}{2} k_{x}+\frac{\sqrt{3}}{2} k_{y}\right)\right], \\
& G_{ \pm r}(\mathbf{k})=2\left[\cos \left(k_{x}\right)+\mathrm{e}^{ \pm 2 \mathrm{i} r \pi / 3} \cos \left(\frac{1}{2} k_{x}+\frac{\sqrt{3}}{2} k_{y}\right)+\mathrm{e}^{ \pm 4 \mathrm{i} r \pi / 3} \cos \left(-\frac{1}{2} k_{x}+\frac{\sqrt{3}}{2} k_{y}\right)\right] .
\end{aligned}
$$

The functions $-\left|G_{2}(\mathbf{k})\right|$ and $G(\mathbf{k})-\left|G_{2}(\mathbf{k})\right|$ are plotted in Fig. 15 and are found to have global minima at $\mathbf{k}_{j}=R_{\psi_{j}} \mathbf{k}_{\mathrm{c}}$ with $\mathbf{k}_{\mathrm{c}}=(0,2 \pi / \sqrt{3})$. Moreover, $F\left(\mathbf{k}_{\mathrm{c}}\right)=1$ so that $\phi_{\mathrm{c}}=0$. Thus the odd and even modes are

$$
\begin{aligned}
& u_{-}(\ell, \theta, \phi)=\left[\sum_{j=1}^{3} u_{j} \sin (2[\phi-2 \pi(j-1) / 3]) \mathrm{e}^{\mathrm{i} \mathbf{k}_{j} \cdot \ell}+\text { c.c. }\right] \sin \theta, \\
& u_{+}(\ell, \theta, \phi)=\left[\sum_{j=1}^{3} u_{j} \cos (2[\phi-2 \pi(j-1) / 3]) \mathrm{e}^{\mathrm{i} \mathbf{k}_{j} \cdot \ell}+\text { c.c. }\right] \sin \theta .
\end{aligned}
$$

In Fig. 16, we show examples of even and odd hexagonal patterns corresponding to the particular choice of amplitudes $u_{j}=1$ for $j=1,2,3$. Again these pictures are constructed using a winner-take-all rule in which only the orientation
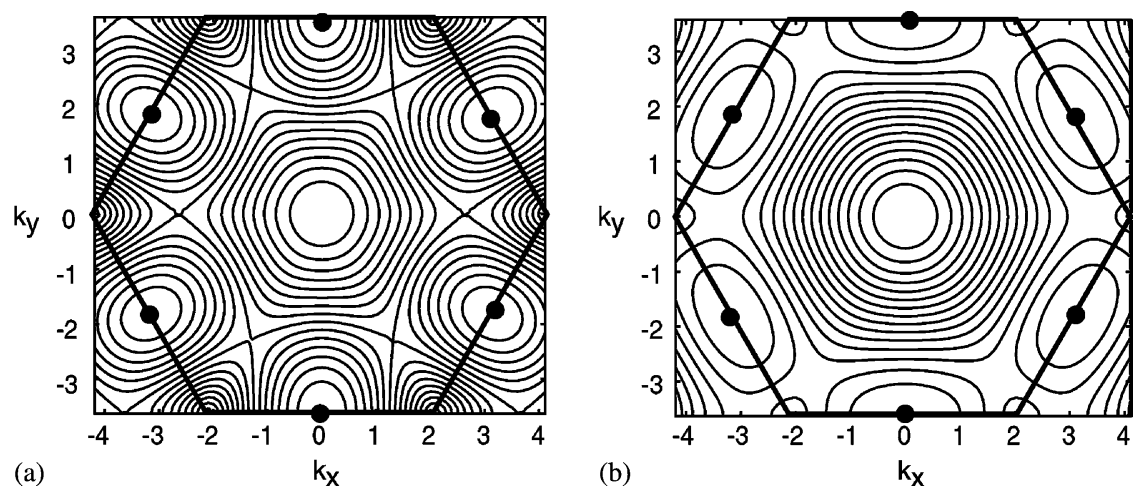

Fig. 15. Contour plots of $-\left|G_{2}(\mathbf{k})\right|$ (a) and $G(\mathbf{k})-\left|G_{2}(\mathbf{k})\right|$ (b) for nearest neighbour coupling on a hexagonal lattice with $\mathbf{k}=\left(k_{x}, k_{y}\right)$ in the first Brillouin zone of the square lattice. Filled circles indicate locations of global minima. 


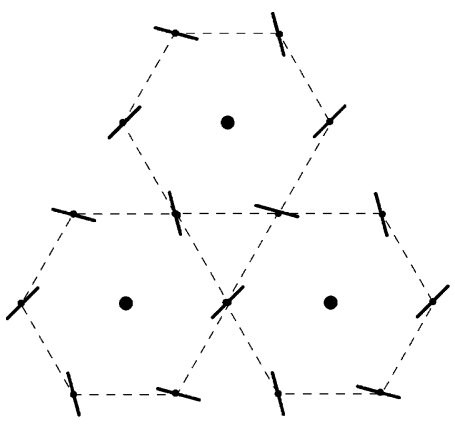

(odd hexagon)

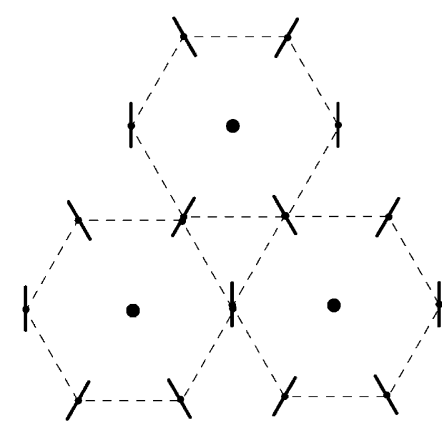

(even hexagon)

Fig. 16. Odd and even hexagonal patterns on a hexagonal lattice with nearest neighbour lateral coupling and amplitude $\mathbf{u}=(1,1,1)$.

of maximal response within each hypercolumn on the lattice is shown. Note, however, that the lattice point at the center of the domain does not show any orientation preference, since there is exact cancellation of the $\phi$-dependent sinusoids in the activity profile of this hypercolumn.

\subsubsection{Inhomogeneous case}

Now suppose that $\chi(\theta)=2 \pi\left(1+\chi_{0} \cos \theta\right)$ with $0<\chi_{0}<1$, representing a coupling that decreases monotonically as the spatial frequency increases [50]. Then $b=1+3 \chi_{0} / 4$ and $b_{0}=1$. Whenever there is an asymmetry between low and high spatial frequencies, the coefficient $b_{1}$ will usually be non-zero. This then generates a mixed marginally stable mode that is a linear combination of Eqs. (4.41), (4.43) and (4.52). However, provided that the asymmetry is not too strong, $b_{1}$ will still be sufficiently small such that mixing is not significant. Following the analysis of the previous example, we find that for sufficiently large $\chi_{0}$

$$
\min _{\mathbf{k}}\left\{\lambda_{0}^{(1)}(\mathbf{k})\right\}<\min _{\mathbf{k}}\left\{\lambda_{-}^{(1)}(\mathbf{k}), \lambda_{+}^{(1)}(\mathbf{k})\right\}
$$

and the marginally stables modes are independent of the orientation label, taking the form

$$
u(\ell, \theta)=C(\ell) \cos \theta
$$

with $C(\ell)$ defined in Eq. (4.11). The peak of the tuning surface alternates as a function of cortical position $\ell$ between the low and high spatial frequency pinwheels, depending on the sign of $C(\ell)$. In Fig. 17, we show some examples of

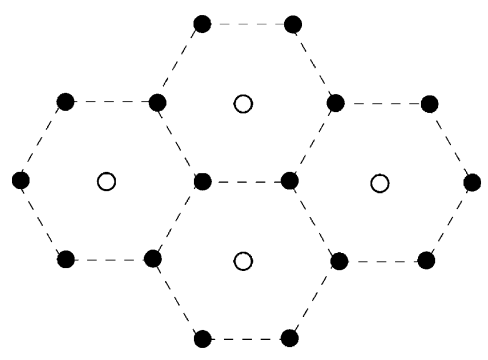

$(1,1,1)$ hexagon

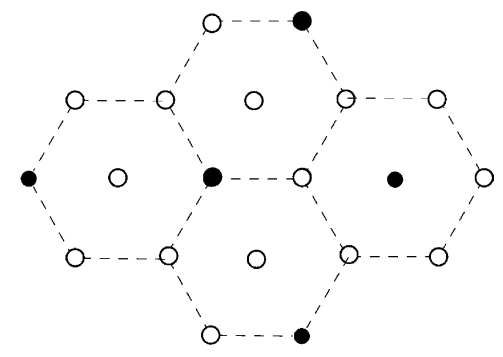

$(1,1,-1)$ hexagon

Fig. 17. Non-contoured hexagonal patterns with high and low spatial frequencies represented by black and white discs: (a) $\mathbf{u}=(1,1,1)$, (b) $\mathbf{u}=(1,1,-1)$. 
hexagonal patterns in which the state of each hypercolumn is reduced to a binary representation indicating whether the maximal response is at the high or low spatial frequency pinwheel.

\subsection{Selection and stability of patterns}

It is necessary to go beyond linear analysis to investigate the selection and stability of the patterns that bifurcate from the homogeneous fixed point. Suppose that in the absence of lateral interactions each hypercolumn destabilises at the critical point $\mu_{\mathrm{c}}=1 / \tilde{W}_{1}$ due to excitation of the first order spherical harmonics (as detailed in Section 3 ). Sufficiently close to the bifurcation point, the resulting cortical state $u=a-\bar{a}$ can be written as

$$
u(\ell, \theta, \phi, t)=\sum_{m=0, \pm 1} z_{m}(\ell, t) Y_{1}^{m}(\theta, \phi)
$$

with $z_{-1}=z_{1}^{*}$. Since the coupling strength $\beta$ of the lateral connections is small, we can carry out a pertubation expansion of Eq. (3.2) with respect to $\beta$ by setting $\mu-\mu_{\mathrm{c}}=\mathcal{O}(\beta)$. As we show in Appendix B, this leads to a cubic amplitude equation for the space dependent coefficients $z_{m}(\ell, t)$ :

$$
\begin{aligned}
\frac{\mathrm{d} z_{m}(\ell, t)}{\mathrm{d} t}= & z_{m}(\boldsymbol{\ell}, t)\left(-1+\mu \tilde{W}_{1}-\frac{4 \pi}{3} \mathcal{W}\left(z_{0}^{2}(\boldsymbol{\ell}, t)+2\left|z_{1}(\boldsymbol{\ell}, t)\right|^{2}\right)\right) \\
& +\beta \sum_{m^{\prime}=0, \pm 1} \sum_{\ell^{\prime} \neq \boldsymbol{\ell}} \int \tilde{J}_{m m^{\prime}}\left(\boldsymbol{\ell}-\boldsymbol{\ell}^{\prime}\right) z_{m^{\prime}}\left(\boldsymbol{\ell}^{\prime}, t\right)
\end{aligned}
$$

where $\mathcal{W}$ is given by Eq. (2.15) and

$$
\sum_{\ell \in \mathcal{L}} \mathrm{e}^{-\mathrm{ik} \cdot \ell} J_{m m^{\prime}}(\ell)=\tilde{J}_{m m^{\prime}}(\mathbf{k})
$$

with $\tilde{J}_{m m^{\prime}}(\mathbf{k})$ defined Eq. (4.21) for $n=n^{\prime}=1$ (assuming anisotropic coupling).

First note that the linearised version of Eq. (4.54) recovers the first order linear dispersion relations derived in Section 4.2. We derive amplitude equations for the corresponding linear eigenmode (4.34) by carrying out a perturbation expansion with respect to a small parameter $\xi$ that determines the distance from the point of marginal stability according to $\xi=\mu-\hat{\mu}_{\mathrm{c}}$ with $\hat{\mu}_{\mathrm{c}}$ given by Eq. (4.33). We introduce a slow time-scale $\tau=\xi^{2} t$ and substitute the series expansion

$$
z_{m}(\ell, \tau)=\xi z_{m}^{(1)}(\ell, \tau)+\xi^{2} z_{m}^{(2)}(\ell, \tau)+\xi^{3} z_{m}^{(3)}(\ell, \tau)+\cdots
$$

into Eq. (4.54). Collecting terms with equal powers of $\xi$ generates a hierarchy of equations of the form (up to $\mathcal{O}\left(\xi^{3}\right)$ )

$$
\begin{aligned}
& \mathcal{M} z_{m}^{(1)}=0, \\
& \mathcal{M} z_{m}^{(2)}=0, \\
& \mathcal{M} z_{m}^{(3)}=z_{m}^{(1)}\left[1-\frac{4 \pi}{3} \mathcal{W}\left(\left|z_{0}^{(1)}\right|^{2}+2\left|z_{1}^{(1)}\right|^{2}\right)\right]-\frac{\mathrm{d} z_{m}^{(1)}}{\mathrm{d} \tau}
\end{aligned}
$$

for $m=0, \pm 1$, where

$$
\mathcal{M} z_{m}(\boldsymbol{\ell}) \equiv-\hat{\mu}_{\mathrm{c}} z_{m}(\boldsymbol{\ell})-\beta \sum_{m^{\prime}=0, \pm 1} \sum_{\boldsymbol{\ell}^{\prime} \neq \boldsymbol{\ell}} \tilde{J}_{m m^{\prime}}\left(\boldsymbol{\ell}-\boldsymbol{\ell}^{\prime}\right) z_{m^{\prime}}\left(\boldsymbol{\ell}^{\prime}\right) .
$$


The first equation in the hierarchy has solutions of the form (4.34):

$$
z_{m}^{(1)}(\ell, \tau)=\sum_{j=1}^{N}\left[U_{m}\left(\mathbf{k}_{j}\right) u_{j}(\tau) \mathrm{e}^{\mathrm{i} \mathbf{k}_{j} \cdot \ell}+U_{-m}^{*}\left(\mathbf{k}_{j}\right) u_{j}^{*}(\tau) \mathrm{e}^{-\mathrm{i} \mathbf{k}_{j} \cdot \ell}\right]
$$

where $N=2$ for the square or rhombic lattice and $N=3$ for the hexagonal lattice. Also $\mathbf{k}_{j}=k_{\mathrm{c}} \hat{\ell}_{j}$ for $j=1,2$ and $\mathbf{k}_{3}=-\mathbf{k}_{1}-\mathbf{k}_{2}$. (By an appropriate phase shift we can take $U_{m}^{*}=U_{m}$.) A dynamical equation for the amplitudes $u_{j}(\tau)$ can then be obtained as a solvability condition for the third order equation (4.59). Define the inner product of two arbitrary functions on the lattice $v_{m}(\ell)$ and $z_{m}(\ell)$ by

$$
\langle v \mid z\rangle=\frac{1}{N^{2}} \sum_{m=0, \pm 1} \sum_{\ell \in \mathcal{L}} v_{m}^{*}(\ell) z_{m}(\ell)
$$

where $N$ determines the size of the lattice, see Eq. (4.4). Taking the inner product of the left-hand side of Eq. (4.59) with $v_{m}(\ell)=U_{m}\left(\mathbf{k}_{j}\right) \mathrm{e}^{\mathrm{i} \mathbf{k}_{j} \cdot \ell}$ leads to the following solvability condition:

$$
\left\langle v \mid \mathcal{M} z^{(3)}\right\rangle=0
$$

Finally, we substitute for $\mathcal{M} z^{(3)}$ using the right-hand side of Eq. (4.59) to obtain an amplitude equation for $u_{j}$ :

$$
\frac{\mathrm{d} u_{j}}{\mathrm{~d} \tau}=u_{j}\left[1-\gamma_{j j}\left|u_{j}\right|^{2}-2 \sum_{l \neq j} \gamma_{j l}\left|u_{l}\right|^{2}\right]
$$

where $\gamma_{j l}=\gamma_{l j}^{(0)}+2 \gamma_{l j}^{(1)}$ with

$$
\gamma_{j l}^{(r)}=\frac{4 \pi \mathcal{W}}{3} \frac{1}{\sum_{m}\left|U_{m}\left(\mathbf{k}_{j}\right)\right|^{2}}\left[\left|U_{r}\left(\mathbf{k}_{j}\right)\right|^{2} \sum_{m}\left|U_{m}\left(\mathbf{k}_{j}\right)\right|^{2}+2 U_{r}^{*}\left(\mathbf{k}_{j}\right) U_{r}\left(\mathbf{k}_{l}\right) \sum_{m} U_{m}^{*}\left(\mathbf{k}_{j}\right) U_{m}\left(\mathbf{k}_{l}\right)\right] .
$$

From the symmetry property (4.35), it can be shown that $\gamma_{j l}$ only depends on the fundamental angle $\Psi$ between the generators of the lattice so that we can write $\gamma_{j l}=\gamma(\Psi)$ for $j \neq l$ and $\gamma_{j j}=\gamma(0)$, where $\gamma$ is a function that will generally depend upon which of the three first order spherical harmonics becomes marginally stable.

Unfortunately, Eq. (4.64) is not sufficient to determine completely the selection and stability of the steady-state solutions bifurcating from the homogeneous state. One has to carry out an unfolding of the amplitude equation, which in the case of the hexagonal lattice includes quadratic as well as higher order terms (quartic and quintic) in $u, u^{*}$. Considerable information about the bifurcating solutions can be obtained using group theoretic methods. In particular, one can use an important result from bifurcation theory in the presence of symmetries, namely, the equivariant branching lemma [24]: when a symmetric dynamical system goes unstable, new solutions emerge that (generically) have symmetries corresponding to the axial subgroups of the underlying symmetry group. A subgroup $\Sigma$ is axial if the dimension of the space of solutions that are fixed by $\Sigma$ is equal to 1 . Thus one can classify the bifurcating solutions by finding the axial subgroups of the symmetry group of the lattice (up to conjugacy). This has been carried out elsewhere for a continuum version of our model (neglecting spatial frequency), with anisotropic lateral connections that are invariant under a shift-twist action of the Euclidean group rather than the discrete lattice group described in Section 3 [11,12]. Restriction to doubly periodic solutions on a planar lattice leads to a bifurcation problem very similar to the one considered in this paper. There is one important difference, however, between the continuum and discrete models that could lead to differences in the axial subgroups. The amplitude equations for doubly periodic solutions of the continuum model are equivariant with respect to the transformation $u_{n} \rightarrow \mathrm{e}^{\mathrm{i} \varphi_{n}} u_{n}$, where $\varphi_{1}, \varphi_{2}$ are arbitrary phases and $\varphi_{3}=-\varphi_{1}-\varphi_{2}$. This reflects the underlying continuous translation symmetry. 
On the other hand, in the lattice model the allowed values of these phases will depend on the particular value of the critical wavenumber $k_{\mathrm{c}}$ and whether or not it is commensurate with the lattice spacing.

\section{Discussion}

Our analysis has shown that in the case of anisotropic lateral connections, there are two very distinct types of pattern that can be generated. The first consists of a contoured pattern in which the optimal response of each hypercolumn occurs at an intermediate spatial frequency and a particular orientation whose direction varies periodically across the lattice as illustrated in Figs. 14 and 16. The second type of pattern is non-contoured and consists of periodic variations in the state of a hypercolumn that alternate between the high and low spatial frequency pinwheels without any particular orientation being preferred, as illustrated in Fig. 17. In the case of nearest neighbour coupling, the critical wavelength of the cortical pattern is equal to half the lattice spacing. However, longer range coupling can lead to other wavelengths as illustrated in Fig. 12(b). Moreover, it is possible for this wavelength to be incommensurate with the lattice so that variations in the state of a hypercolumn are quasi-periodic rather than periodic across the cortex.

Any spontaneously generated or stimulus-evoked cortical activity pattern in V1 maps to a corresponding real or hallucinatory image on the retina [11,21]. In order to reconstruct such an image, it is first necessary to make some ansatz regarding the interpretation of the cortical pattern and then to determine how the interpreted or reduced pattern transforms under the action of the retino-cortical map. One possible interpretation of a contoured cortical pattern is that it corresponds to a vector field of orientations. It is then relatively straightforward to pull back such a vector field to generate a contoured image on the retina [11]. The interpretation of a non-contoured pattern is less clear. One possibility is to pull back low spatial frequency patterns into alternating levels of high and low brightness and to pullback high spatial frequency patterns into textured images.

The important distinction between even or odd contoured images and non-contoured images was previously highlighted in our analysis of a continuum model of cortex, consisting of a continuous two-dimensional sheet of interacting hypercolumns [11,12]. In this earlier model, each hypercolumn was idealised as a ring of orientation selective cells-spatial frequency selectivity was ignored. We showed that the resulting distribution of lateral connections was invariant under a shift-twist action of the planar Euclidean group $\mathbf{E}(2)$ acting on the product space $\mathbf{R}^{2} \times \mathbf{S}^{1}$. By virtue of the anisotropy of the lateral connections, this shift-twist symmetry supported distinct scalar and pseudoscalar group representations of $\mathbf{E}(2)$ [9], which characterised the type of cortical activity patterns that could arise through spontaneous symmetry breaking $[11,12]$. Continuous rotation symmetry implied that the manifold of marginally stable states was infinite dimensional, consisting of spatially periodic patterns with wavectors lying on some critical circle $|\mathbf{k}|=k_{\mathrm{c}}$. In order to carry out a finite-dimensional bifurcation analysis, we followed the usual procedure of restricting solutions to be doubly periodic with respect to some planar lattice whose lattice spacing was taken to be equal to the critical wavelength of the marginally stable solutions. This then led to the discrete shift-twist group action of Eq. (3.8).

Two examples of common hallucinatory images reproduced by our continuum model [11] are shown in Fig. 18. The first is a non-contoured image consisting of alternating regions of high and low luminance. The corresponding cortical states are ones in which individual hypercolumns do not amplify any particular orientation, under the assumption that there is a reduction in local inhibition within a hypercolumn that results in a bulk instability with respect to orientation. On the other hand, the second image is contoured, consisting of a network of locally oriented contrast edges obtained as the pull back of a vector field of optimal orientations in cortex. The inclusion of orientation selective cells into a cortical model of pattern formation thus allows all of the basic types of hallucinatory images (form constants) classified by Klüver [33] to be accounted for [11] - this was not possible in the original model of Ermentrout and Cowan [21], which could only generate non-contoured patterns. 
(a)

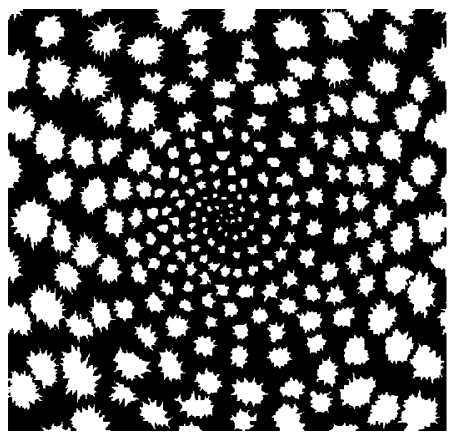

(b)

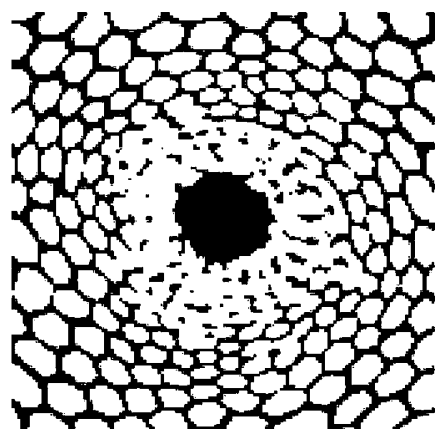

Fig. 18. Non-contoured and contoured hallucinatory images: (a) spiral images seen following ingestion of LSD (redrawn from [43]), (b) honeycomb generated by marihuana (redrawn from [19]).

The model presented in this paper improves upon our continuum model in two important aspects. First, interacting hypercolumns are distributed on a lattice rather than a continuum. This is more realistic from a biological perspective, given the interpretation of a hypercolumn as a fundamental domain of a lattice of orientation pinwheels. It also has the advantage of eliminating certain ad hoc aspects of our previous analysis. In particular, the existence of a physical lattice means that doubly periodic patterns such as rolls, hexagons and squares arise naturally within our theory, rather than being imposed arbitrarily as a mathematical simplification. Moreover, once contoured patterns have been generated in the continuum model, it is necessary to carry out some discrete sampling scheme in order to construct the vector field of orientations using a winner-take-all strategy. In the lattice model, the sampling is already specified by the distribution of pinwheels. Thus, there is a much stronger connection between the types of hallucinatory images generated by our lattice model and the underlying structure of cortex. Second, we have introduced a more detailed model of the internal structure of a hypercolumn that takes into account the spatial frequency selectivity of cortical neurons. This is particularly natural given the observation that the pinwheels are organising centres for both the orientation and spatial frequency maps $[8,32]$. Inclusion of spatial frequency provides an alternative explanation of the origins of contoured versus non-contoured hallucinations, namely, that contoured (non-contoured) hallucinations arise when intermediate (low/high) spatial frequencies within a hypercolumn are excited (as would occur when there is sufficient inhomogeneity in the lateral connections, see Section 4.2). This, in turn, supports the idea that there are at two least distinct circuits operating in V1 [6], one dealing with contrast edges at intermediate spatial frequencies and another, centred on the orientation pinwheels, involved with the processing of textures, surfaces and brightness.

Our new model still involves a number of simplifications. First, there is clearly some degree of disorder in the distribution of orientation pinwheels so that it might be more appropriate to consider a disordered rather than an ordered lattice of hypercolumns. Second, we have carried out a phenomenological reduction of the internal structure of each hypercolumn along the lines illustrated in Fig. 3. Such a reduction needs to be carried out in a more rigorous and systematic fashion, in order to fully account for how the local and long-range connections are correlated with the two-dimensional orientation and spatial frequency maps. Alternatively, one could consider a continuum model of cortex that explicitly incorporates these correlations. A preliminary analysis of such a model suggests that double periodicity in the feature maps can induce an analogue of Bloch waves found in crytalline solids [3], in which activity patterns are localised around pinwheels or around linear zones [13]. Finally, it would be interesting to consider how the periodic structures described in this paper actually develop and subsequently influence the development of other cortical structures. Since many models of activity-based cortical development involve some form of pattern forming instability mediated by lateral connections [46], it is likely that some of the techniques familiar in the study of ordered (and disordered) crystalline structures will also be relevant. 


\section{Acknowledgements}

This work was supported in part by grant 0209824 from the NSF to P.C.B. and by grant $96-24$ from the James S. McDonnell Foundation to J.D.C.

\section{Appendix A}

Here we derive the amplitude equation (2.14) and determine the coefficient $\mathcal{W}$. First, substitute the perturbation series

$$
a=\bar{a}+\varepsilon^{1 / 2} a_{1}+\varepsilon a_{2}+\varepsilon^{3 / 2} a_{3}+\cdots
$$

into Eq. (2.2) with $a_{m}=a_{m}(\theta, \phi, t)$ and Taylor expand the nonlinear firing rate function using (2.16). Introduce a slow time scale $\tau=\varepsilon t$ and collect terms with equal powers of $\varepsilon$. This leads to a hierarchy of equations of the form

$$
\begin{aligned}
& \hat{L} a_{1}=0, \\
& \hat{L} a_{2}=h_{2}\left[a_{1}\right] \equiv \sigma_{2} \mu_{\mathrm{c}} W * a_{1}^{2}, \\
& \hat{L} a_{3}=h_{3}\left[a_{1}, a_{2}\right] \equiv \sigma_{3} \mu_{\mathrm{c}} W * a_{1}^{3}+2 \sigma_{2} \mu_{\mathrm{c}} W * a_{1} a_{2}+\mu_{\mathrm{c}}^{-1} a_{1}-\frac{\partial a_{1}}{\partial \tau},
\end{aligned}
$$

where

$$
\hat{L} a=a-\mu_{\mathrm{c}} W * a
$$

and, for any function $a: S^{2} \rightarrow \mathbf{R}$,

$$
[W * a](\theta, \phi)=2 \int_{0}^{\pi} \int_{0}^{\pi} \sum_{n=0}^{\infty} \tilde{W}_{n} \sum_{m=-n}^{n} Y_{n}^{m^{*}}\left(\theta^{\prime}, \phi^{\prime}\right) Y_{n}^{m}(\theta, \phi) a\left(\theta^{\prime}, \phi^{\prime}\right) \sin \theta^{\prime} \mathrm{d} \theta^{\prime} \mathrm{d} \phi^{\prime} .
$$

The lowest order equation (A.2) has solutions of the form

$$
a_{1}(\theta, \phi, \tau)=\sum_{m=0, \pm 1} c_{m}(\tau) f_{m}(\theta, \phi)
$$

with $f_{m}$ defined in Eq. (2.13). The higher order equations, on the other hand, only have solutions if certain solvability conditions are satisfied. Define the inner product of two functions $U, V: S^{2} \rightarrow \mathbf{R}$ according to

$$
\langle U \mid V\rangle=2 \int_{0}^{\pi} \int_{0}^{\pi} U^{*}(\theta, \phi) V(\theta, \phi) \sin (\theta) \mathrm{d} \theta \mathrm{d} \phi .
$$

The linear operator is then self adjoint so that taking the inner product of Eqs. (A.3) and (A.4) with the functions $f_{m}$ (which are linear combinations of the first order spherical harmonics) leads to the result

$$
\left\langle f_{m} \mid h_{k}\right\rangle=\left\langle f_{m} \mid \hat{L} a_{k}\right\rangle=\left\langle\hat{L} f_{m} \mid a_{k}\right\rangle=0
$$

for $k=2,3$ and $m=0, \pm 1$. The solvability condition $\left\langle f_{m} \mid h_{2}\right\rangle=0$ is automatically satisfied since $\left\langle f_{m} \mid W * a_{1}^{2}\right\rangle=$ $\mu_{\mathrm{c}}^{-1}\left\langle f_{m} \mid a_{1}^{2}\right\rangle=0$. Therefore, we focus on the cubic solvability condition $\left\langle f_{m} \mid h_{3}\right\rangle=0$, which reduces to the form

$$
\left\langle f_{m} \mid \frac{\partial a_{1}}{\partial \tau}-\mu_{\mathrm{c}}^{-1} a_{1}\right\rangle=\sigma_{3}\left\langle f_{m} \mid a_{1}^{3}\right\rangle+2 \sigma_{2}\left\langle f_{m} \mid a_{1} a_{2}\right\rangle, \quad m=0, \pm 1 .
$$

We evaluate the various inner products appearing in Eq. (A.10) for $m=0$. This will generate a cubic amplitude equation for $c_{0}$ : the other amplitude equations follow immediately from the underlying $\mathbf{O}(3)$ symmetry. 
First, from Eq. (A.7) we have

$$
\left\langle\cos \theta \mid \frac{\partial a_{1}}{\partial \tau}-\mu_{\mathrm{c}}^{-1} a_{1}\right\rangle=\left(\frac{\mathrm{d} c_{0}}{\mathrm{~d} \tau}-\mu_{\mathrm{c}}^{-1} c_{0}\right)\langle\cos \theta \mid \cos \theta\rangle=\frac{4 \pi}{3}\left(\frac{\mathrm{d} c_{0}}{\mathrm{~d} \tau}-\mu_{\mathrm{c}}^{-1} c_{0}\right) .
$$

Next, expand $a_{1}^{3}$ and collect terms that are independent of $\phi$ :

$$
\left\langle\cos \theta \mid a_{1}^{3}\right\rangle=c_{0}^{3}\left\langle\cos \theta \mid \cos ^{3} \theta\right\rangle+\frac{3}{2} c_{0}\left(c_{1}^{2}+c_{-1}^{2}\right)\left\langle\cos \theta \mid \cos \theta \sin ^{2} \theta\right\rangle=\frac{4 \pi}{5} c_{0} \sum_{m=0, \pm 1} c_{m}^{2} .
$$

In order to evaluate the final term on the right-hand side of Eq. (A.10), we solve for $a_{2}$ in terms of $a_{1}$ using Eq. (A.3). Decompose the product $a_{1}^{2}$ as a sum of zeroth and second order harmonics (see Table 1) so that

$$
\begin{aligned}
a_{2}-\mu_{\mathrm{c}} W * a_{2}= & \frac{1}{3} \sigma_{2} \mu_{\mathrm{c}} \tilde{W}_{0} \sum_{m=0, \pm 1} c_{m}^{2}+\sigma_{2} \mu_{\mathrm{c}} \tilde{W}_{2}\left[\frac{c_{+}^{2}-c_{-}^{2}}{2} \sin ^{2} \theta \cos 4 \phi+c_{1} c_{-1} \sin ^{2} \theta \sin 4 \phi\right] \\
& +\sigma_{2} \mu_{\mathrm{c}} \tilde{W}_{2} c_{0} \sin 2 \theta\left[c_{1} \cos 2 \phi+c_{-1} \sin 2 \phi\right]+\sigma_{2} \mu_{\mathrm{c}} \tilde{W}_{2}\left(\frac{1}{3}-\cos ^{2} \theta\right)\left(\frac{c_{1}^{2}+c_{-1}^{2}}{2}-c_{0}^{2}\right) .
\end{aligned}
$$

It follows that

$$
\begin{aligned}
a_{2}= & \kappa a_{1}(\theta, \phi)+\frac{\Lambda_{0}}{3} \sum_{m=0, \pm 1} c_{m}^{2}+\Lambda_{2}\left[\frac{c_{1}^{2}-c_{-1}^{2}}{2} \sin ^{2} \theta \cos 4 \phi+c_{1} c_{-1} \sin ^{2} \theta \sin 4 \phi\right] \\
& +\Lambda_{2}\left[c_{0} \sin 2 \theta\left[c_{1} \cos 2 \phi+c_{-1} \sin 2 \phi\right]+\left(\frac{1}{3}-\cos ^{2} \theta\right)\left(\frac{c_{1}^{2}+c_{-1}^{2}}{2}-c_{0}^{2}\right)\right],
\end{aligned}
$$

where

$$
\Lambda_{r}=\frac{\sigma_{2} \mu_{\mathrm{c}} \tilde{W}_{r}}{1-\mu_{\mathrm{c}} \tilde{W}_{r}}, \quad r=0,2 .
$$

The constant $\kappa$ remains undetermined at this level of perturbation analysis, but does not contribute to the cubic amplitude equation for $c_{0}, c_{ \pm}$. We now use Eq. (A.14) to expand the product $a_{2} a_{1}$. Keeping only $\phi$-independent terms then gives

$$
\begin{aligned}
\left\langle\cos \theta \mid a_{1} a_{2}\right\rangle= & \frac{\Lambda_{0}}{3}\left[c_{0} \sum_{m=0, \pm 1} c_{m}^{2}\right]\langle\cos \theta \mid \cos \theta\rangle+\frac{\Lambda_{2}}{2} c_{0}\left[c_{1}^{2}+c_{-1}^{2}\right]\langle\cos \theta \mid \sin \theta \sin 2 \theta\rangle \\
& +\frac{\Lambda_{2}}{2} c_{0}\left(c_{1}^{2}+c_{-1}^{2}-2 c_{0}^{2}\right)\left\langle\cos \theta \mid \frac{\cos \theta}{3}-\cos ^{3} \theta\right\rangle .
\end{aligned}
$$

Evaluating the various inner products using Eq. (A.8) yields the result

$$
\left\langle\cos \theta \mid a_{1} a_{2}\right\rangle=\left[\frac{4 \pi}{3} \frac{\Lambda_{0}}{3}+\frac{32 \pi}{45} \frac{\Lambda_{2}}{2}\right]\left[c_{0} \sum_{m=0, \pm 1} c_{m}^{2}\right] .
$$

The final step in the analysis is to substitute Eqs. (A.11), (A.12) and (A.17) into Eq. (A.10) for $m=0$. This gives

$$
\frac{\mathrm{d} c_{m}}{\mathrm{~d} \tau}=c_{m}\left(\mu_{\mathrm{c}}^{-1}-\mathcal{W} \sum_{p=0, \pm 1} c_{p}^{2}\right)
$$

for $m=0$ with the coefficient $\mathcal{W}$ satisfying Eq. (2.15). After rescaling $c_{m}$ and $\tau$, we recover Eq. (2.14). 


\section{Appendix B}

We sketch how to extend the amplitude equation analysis of Appendix A to the coupled hypercolumn model of Section 4. Exploiting the fact that the horizontal interactions are weak, we assume that each active hypercolumn is $\varepsilon$-close to bifurcation and $\beta=\mathcal{O}(\varepsilon)$. Carrying out a perturbation expansion of Eq. (3.2) using Eq. (A.1) with $a_{m}=a_{m}(\ell, \theta, \phi, \tau)$ leads to the hierarchy of Eqs. (A.2)-(A.4), except that now there are additional contributions arising from the lateral interactions:

$$
\begin{aligned}
& \hat{L} a_{1}=0, \\
& \hat{L} a_{2}=\sigma_{2} \mu_{\mathrm{c}} W *\left(a_{1}\right)^{2}, \\
& \hat{L} a_{3}=\sigma_{3} \mu_{\mathrm{c}} W *\left(a_{1}\right)^{3}+2 \sigma_{2} \mu_{\mathrm{c}} W * a_{1} a_{2}+\mu_{\mathrm{c}}^{-1} a_{1}-\frac{\partial a_{1}}{\partial \tau}+\hat{\beta} w_{\text {lat }} \circ a_{1},
\end{aligned}
$$

where $\beta=\hat{\beta} \varepsilon$. Here $\hat{L}$ is the linear operator (A.5), $W * A$ is defined in Eq. (A.6) and

$$
\left[w_{\text {lat }} \circ a\right](\ell, \theta, \phi)=\sum_{\ell^{\prime} \neq \ell} J\left(\ell-\ell^{\prime}\right) \int \mathcal{A}_{\ell, \ell^{\prime}}\left(P^{\prime}\right) a\left(\ell^{\prime}, P^{\prime}\right) D P^{\prime} .
$$

The lowest order equation (B.1) has solutions of the form

$$
a_{1}(\ell, \theta, \phi, \tau)=\sum_{m=0, \pm 1} z_{m}(\ell, \tau) Y_{1}^{m}(\theta, \phi) .
$$

(Note that in the lattice model, it is more convenient to work with the complex basis $Y_{1}^{m}$ for the spherical harmonics.) As in Appendix A, dynamical equations for the amplitudes $z_{m}$ can be derived from the solvability conditions obtained by taking the inner product of the third order equation with respect to $Y_{1}^{m}$. This gives

$$
\left\langle Y_{1}^{m} \mid \frac{\partial a_{1}}{\partial \tau}-\mu_{\mathrm{c}}^{-1} a_{1}\right\rangle=\sigma_{3}\left\langle Y_{1}^{m} \mid\left(a_{1}\right)^{3}\right\rangle+2 \sigma_{2}\left\langle Y_{1}^{m} \mid a_{1} a_{2}\right\rangle+\hat{\beta}\left\langle Y_{1}^{m} \mid w_{\text {lat }} \circ a_{1}\right\rangle
$$

for $m=0, \pm 1$.

Finally, substitute Eq. (B.5) into (B.6) and evaluate the inner products. The local contributions to the complex amplitude equation are obtained from Eq. (A.18) by performing the coordinate transformation

$$
z_{0}=\sqrt{\frac{3}{4 \pi}} c_{0}, \quad z_{ \pm 1}=\sqrt{\frac{3}{8 \pi}}\left(c_{1} \pm \mathrm{i} c_{-1}\right) .
$$

Thus

$$
\frac{\mathrm{d} z_{m}}{\mathrm{~d} \tau}=z_{m}\left(\mu_{\mathrm{c}}^{-1}-\frac{4 \pi}{3} \mathcal{W}\left(z_{0}^{2}+2\left|z_{1}\right|^{2}\right)\right)+\hat{\beta}\left\langle Y_{1}^{m} \mid w_{\text {lat }} \circ a_{1}\right\rangle .
$$

The interaction term $\left\langle Y_{1}^{m} \mid w_{\text {lat }} \circ a_{1}\right\rangle$ is most easily calculated using Fourier transforms. Following the analysis of Section 4 , we find that

$$
w_{\text {lat }} \circ a_{1}(\ell, \theta, \phi, \tau)=\frac{L^{2}}{4 \pi^{2}} \sum_{m^{\prime}=0, \pm 1}\left[\int \mathrm{e}^{\mathrm{i} \mathbf{k} \cdot \ell} \tilde{J}(\mathbf{k} \mid \theta, \phi) Z_{m^{\prime}}(\mathbf{k}, \tau) \mathrm{d}^{2} \mathbf{k}\right] Y_{1}^{m^{\prime}}(\theta, \phi),
$$

where $\tilde{J}(\mathbf{k} \mid \theta, \phi)$ is given by Eq. (4.17) and $Z_{m}(\mathbf{k}, \tau)$ is the Fourier transform of $z_{m}(\ell, \tau)$. Hence,

$$
\left\langle Y_{1}^{m} \mid w_{\text {lat }} \circ a_{1}\right\rangle=\frac{L^{2}}{4 \pi^{2}} \sum_{m^{\prime}=0, \pm 1}\left[\int \mathrm{e}^{\mathrm{ik} \cdot \ell} \tilde{J}_{m m^{\prime}}(\mathbf{k}) Z_{m^{\prime}}(\mathbf{k}, t) \mathrm{d}^{2} \mathbf{k}\right]
$$


with $\tilde{J}_{m m^{\prime}}(\mathbf{k})$ defined by Eq. (4.21) for $n=n^{\prime}=1$. After rescaling $z_{m}$ and $\tau$, and using the convolution theorem we obtain the cubic amplitude equation (4.54) for the lattice model.

\section{References}

[1] A. Angelucci, J.B. Levitt, E.J.S. Walton, J.-M. Hupé, J. Bullier, J.S. Lund, Circuits for local and global signal integration in primary visual cortex. J. Neurosci. 22 (2002) 8633-8646.

[2] G. Arfken, Mathematical Methods for Physicists, 3rd ed., Academic Press, San Diego, 1985.

[3] N.W. Ashcroft, N.D. Mermin, Solid State Physics, Saunders, London, 1976.

[4] R. Ben-Yishai, R. Lev Bar-Or, H. Sompolinsky, Theory of orientation tuning in visual cortex, Proc. Natl. Acad. Sci. 92 (1995) $3844-3848$.

[5] G.G. Blasdel, G. Salama, Voltage-sensitive dyes reveal a modular organization in monkey striate cortex, Nature 321 (1986) $579-585$.

[6] G.G. Blasdel, Orientation selectivity, preference, and continuity in monkey striate cortex, J. Neurosci. 12 (1992) $3139-3161$.

[7] T. Bonhoeffer, A. Grinvald, Orientation columns in cat are organized in pinwheel like patterns, Nature 364 (1991) $146-166$.

[8] T. Bonhoeffer, D.S. Kim, D. Malonek, D. Shoham, A. Grinvald, Optical Imaging of the layout of functional domains in area $17 / 18$ border in cat visual cortex, Eur. J. Neurosci. 7 (9) (1995) 1973-1988.

[9] I. Bosch Vivancos, P. Chossat, I. Melbourne, New planforms in systems of partial differential equations with Euclidean symmetry, Arch. Rat. Mech. 131 (1995) 199-224.

[10] W.H. Bosking, Y. Zhang, B. Schofield, D. Fitzpatrick, Orientation selectivity and the arrangement of horizontal connections in tree shrew striate cortex, J. Neurosci. 17 (1997) 2112-2127.

[11] P.C. Bressloff, J.D. Cowan, M. Golubitsky, P.J. Thomas, M. Wiener, Geometric visual hallucinations, Euclidean symmetry and the functional architecture of striate cortex, Phil. Trans. R. Soc. Lond. B 356 (2001) 299-330.

[12] P.C. Bressloff, J.D. Cowan, M. Golubitsky, P.J. Thomas, Scalar and pseudoscalar bifurcations: pattern formation on the visual cortex, Nonlinearity 14 (2001) 739-775.

[13] P.C. Bressloff, Bloch waves, periodic feature maps and cortical pattern formation, Phys. Rev. Lett. 89 (2002) 088101.

[14] P.C. Bressloff, J.D. Cowan, An SO(3) symmetry breaking mechanism for orientation and spatial frequency tuning in visual cortex, Phys. Rev. Lett. 88 (2002) 078102.

[15] P.C. Bressloff, J.D. Cowan, A spherical model of orientation and spatial frequency tuning in a cortical hypercolumn, Phil. Trans. Roy. Soc. $\mathrm{B}$, in press.

[16] P.C. Bressloff, J.D. Cowan, Spontaneous pattern formation in primary visual cortex, in: S.J. Hogan, A. Champneys, B. Krauskopf (Eds.), Nonlinear Dynamics and Chaos: Where do We Go from Here? Institute of Physics, Bristol, Chapter 11.

[17] P.C. Bressloff, J.D. Cowan, M. Golubitsky, P.J. Thomas, M. Wiener, What geometric visual hallucinations tell us about the visual cortex? Neural Comput. 14 (2002) 471-492.

[18] P.C. Bressloff, J.D. Cowan, An amplitude equation approach to contextual effects in primary visual cortex, Neural Comput. 14 (2002) 493-525.

[19] J. Clottes, D. Lewis-Williams, The Shamans of Prehistory: Trance and Magic in the Painted Caves, Abrams, New York, 1998.

[20] R.J. Douglas, C. Koch, M. Mahowald, K.A.C. Martin, H.H. Suarez, Recurrent excitation in neocortical circuits, Science 269 (1995) 981-985.

[21] G.B. Ermentrout, J.D. Cowan, A mathematical theory of visual hallucination patterns, Biol. Cybern. 34 (1979) 137-150.

[22] G.B. Ermentrout, Neural networks as spatial pattern forming systems, Rep. Prog. Phys. 61 (1998) 353-430.

[23] C.D. Gilbert, T.N. Wiesel, Clustered intrinsic connections in cat visual cortex, J. Neurosci. 3 (1983) 1116-1133.

[24] M. Golubitsky, I. Stewart, D.G. Schaeffer, Singularities and Groups in Bifurcation Theory II, Springer, Berlin, 1988.

[25] M.J. Hawken, A.J. Parker, Spatial properties of neurons in the monkey striate cortex, Proc. R. Soc. B 231 (1987) $251-288$.

[26] J.D. Hirsch, C.D. Gilbert, Synaptic physiology of horizontal connections in the cat's visual cortex, J. Physiol. Lond. 160 (1992) $106-154$.

[27] D.H. Hubel, T.N. Wiesel, Receptive fields, binocular interaction and functional architecture in the cat's visual cortex, J. Neurosci. 3 (1962) $1116-1133$.

[28] D.H. Hubel, T.N. Wiesel, Receptive fields and functional architecture of monkey striate cortex, J. Physiol. Lond. 195 (1968) $215-243$.

[29] D.H. Hubel, T.N. Wiesel, Uniformity of monkey striate cortex: a parallel relationship between field size, scatter, and magnification factor, J. Comput. Neurol. 158 (1974) 295-306.

[30] D.H. Hubel, T.N. Wiesel, Functional architecture of macaque monkey visual cortex, Proc. R. Soc. B 198 (1977) 1-59.

[31] M. Hübener, D. Shoham, A. Grinvald, T. Bonhoeffer, Spatial relationships among three columnar systems in cat area 17, J. Neurosci. 17 (23) (1997) 9270-9284.

[32] N.P. Issa, C. Trepel, M.P. Stryker, Spatial frequency maps in cat visual cortex, J. Neurosci. 20 (2000) 8504-8514.

[33] H. Klüver, Mescal and Mechanisms of Hallucinations, University of Chicago Press, Chicago, 1966.

[34] S. LeVay, S.B. Nelson, Columnar organization of the visual cortex, in: A.G. Leventhal (Ed.), The Neural Basis of Visual Function, CRC Press, Boca Raton, FL, 1991, pp. 266-315.

[35] M.S. Livingstone, D.H. Hubel, Specificity of intrinsic connections in primate primary visual cortex, J. Neurosci. 4 (1984) $2830-2835$.

[36] R. Malach, Y. Amir, M. Harel, A. Grinvald, Relationship between intrinsic connections and functional architecture revealed by optical imaging and in vivo targeted biocytin injections in primate striate cortex, Proc. Natl. Acad. Sci. 90 (1993) 10469-10473. 
[37] P.E. Maldonado, I. Gödecke, C.M. Gray, T. Bonhoeffer, Orientation selectivity in pinwheel centers in cat striate cortex, Science 276 (1997) 1551-1555.

[38] J.A. Mazer, W.E. Vinje, J. McDermott, P.H. Schiller, J.L. Gallant, Spatial frequency and orientation tuning dynamics in V1, Proc. Natl. Acad. Sci. 99 (2002) 1645-1650.

[39] L.P. O’ Keefe, J.B. Levitt, D.C. Kiper, R.M. Shapley, J.A. Movshon, Functional organization of owl monkey lateral geniculate nucleus and visual cortex, J. Neurophysiol. 80 (1998) 594-609.

[40] K. Obermayer, G. Blasdel, Geometry of orientation and ocular dominance columns in monkey striate cortex, J. Neurosci. 13 (1993) 4114-4129.

[41] K.S. Rockland, J.S. Lund, Intrinsic laminar lattice connections in primate visual cortex, J. Comput. Neurol. 216 (1983) $303-318$.

[42] B. Roerig, B. Chen, Relationships of local inhibitory and excitatory circuits to orientation preference maps in ferret visual cortex, Cereb. Cortex 12 (2002) 187-198.

[43] R.K. Siegel, M.E. Jarvik, Drug-induced hallucinations in animals and man, in: R.K. Siegel, L.J. West (Eds.), Hallucinations: Behavior, Experience and Theory, Wiley, New York, 1975, pp. 81-161.

[44] L.C. Sincich, G.G. Blasdel, Oriented axon projections in primary visual cortex of the monkey, J. Neurosci. 21 (2001) $4416-4426$.

[45] D.C. Somers, S. Nelson, M. Sur, An emergent model of orientation selectivity in cat visual cortical simple cells, J. Neurosci. 15 (1995) 5448-5465.

[46] N.V. Swindale, The development of topography in visual cortex: a review of models, Network 7 (1996) 161-247.

[47] A.M. Turing, The chemical basis of morphogenesis, Phil. Trans. R Soc. Lond. B 237 (1952) 32-72.

[48] C. Varea, J.L. Aragon, R.A. Barrio, Turing patterns on a sphere, Phys. Rev. E 60 (10) (1999) 4588-4592.

[49] T. Yoshioka, G.G. Blasdel, J.B. Levitt, J.S. Lund, Relation between patterns of intrinsic lateral connectivity, ocular dominance, and cytochrome oxidase-reactive regions in macaque monkey striate cortex, Cereb. Cortex 6 (1996) 297-310.

[50] N.H. Yabuta, E.M. Callaway, Cytochrome-oxidase blobs and intrinsic horizontal connections of layer $2 / 3$ pyramidal neurons in primate V1, Vis. Neurosci. 15 (1998) 1007-1027. 